\title{
A Transferable Model for Adsorption in MOFs with Unsaturated Metal Sites
}

\author{
Christopher Campbell, ${ }^{*}{ }^{\dagger}$ Carlos A. Ferreiro-Rangel, ${ }^{\dagger}$ Michael Fischer, ${ }^{\ddagger}, \S$ José R. B. Gomes, ${ }^{\|}$ \\ and Miguel Jorge ${ }^{*}, \dagger$ \\ ${ }^{\dagger}$ Department of Chemical and Process Engineering, University of Strathclyde, 75 Montrose Street, Glasgow, Scotland G1 1XJ, United \\ Kingdom \\ ${ }^{\ddagger}$ Crystallography Group, Department of Geosciences, University of Bremen, Klagenfurter Straße, 28359 Bremen, Germany \\ ${ }^{\S}$ MAPEX Center for Materials and Processes, 28359 Bremen, Germany \\ "CICECO-Aveiro Institute of Materials, Department of Chemistry, University of Aveiro, Campus Universitário de Santiago, 3810-193 \\ Aveiro, Portugal
}

\section{Supporting Information}

ABSTRACT: The number of newly discovered metalorganic frameworks is growing exponentially. Molecular simulation is becoming increasingly important to screen large databases of structures and identify potential candidates for challenging gas separations, but such efforts rely on the availability of accurate molecular models that can predict adsorption in a wide range of different MOFs. MOFs with coordinatively unsaturated sites (CUS) pose particular problems because standard force fields are unable to describe
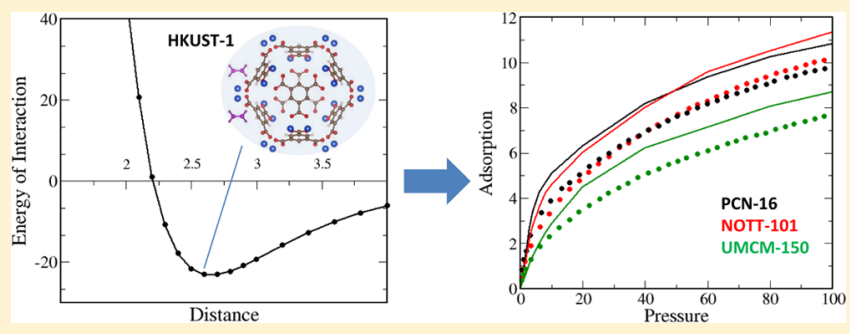
their specific interactions with certain adsorbates. In this Article, we demonstrate that our previous approach to describe adsorption in open metal sites, based on a combination of classical Monte Carlo simulations and quantum-mechanical Density Functional Theory calculations, is transferable to several Cu-containing MOFs. By fitting the parameters of our model to match adsorption energies of ethylene on HKUST-1 and transferring them to the Cu-paddlewheel units of other MOFs, we obtain predictions in good agreement with experimental adsorption measurements. Where agreement is not as satisfactory, we show that this can be explained by limited accessibility or diffusion through the pore network. For one particular MOF, UMCM-150, we show that separate parameters need to be used for the Cu-trimer unit, for which the interaction energies with ethylene are much lower than those in the Cu-paddlewheel. Overall, our approach demonstrates that the specific CUS interactions in MOFs can be parametrized separately from other interaction types, such as van der Waals, thus opening the way for the development of an accurate and fully transferable force field for this class of materials.

\section{INTRODUCTION}

The research into metal-organic frameworks (MOFs) as materials for adsorption separation processes has gained increasing interest within the scientific community in recent years. ${ }^{1}$ These porous materials are made up of organic linkers and metal centers that can be combined into three-dimensional nanoporous polymer frameworks. The interest in these materials is primarily rooted in their very high attainable porosities and in their tailorability, derived from the various possible choices of ligands and metals that can create a variety of topologies and adsorption sites. The sheer number of MOF variations becomes apparent when looking at the Cambridge Structural Database, which currently contains 50000 MOF structures. $^{2}$ This number is expected to continue to grow rapidly with the development of computational software designed to identify potentially undiscovered MOFs. ${ }^{3,4}$ This variability provides the opportunity to specifically tailor MOFs to particular adsorption-based applications. For example, it has been shown that their organic ligands can be functionalized with amines, thus increasing the affinity of the MOF for carbon dioxide. ${ }^{5}$ Additionally, through techniques such as linker choice $^{6}$ and interpenetration, 7 the physical pore sizes of MOFs can be varied, thereby enabling increased selectivity through control of the adsorbate kinetics. Nugent et al. ${ }^{7}$ used this technique of controlled functionalization on SIFSIX MOFs, optimizing kinetic and thermodynamic properties for selective adsorption of carbon dioxide from various gas mixtures.

This attractive feature also has the caveat that experimental analysis of even just one single MOF family of materials for a given application is challenging due to the time-consuming nature of experiments. It is in this initial analysis that molecular simulation can play an important role by narrowing the field and highlighting the best potential MOFs for detailed experimental analysis. Indeed, some recent works aimed to

Received: October 25, 2016

Revised: December 7, 2016

Published: December 8, 2016 
use large-scale computational screening to identify promising MOFs for adsorption-based applications. In the work by Wilmer et al., ${ }^{4}$ a building-block method based on existing MOF structures was utilized to identify 130000 potential MOFs. The authors then went on to computationally analyze physical characteristics of the MOFs as well as methane uptake capacities, which were adequately confirmed through experiment for some of the highest performing MOFs. High performance screening conducted by Watanabe et al. ${ }^{8}$ also managed to examine $30000 \mathrm{MOF}$ to evaluate the potential for $\mathrm{CO}_{2} / \mathrm{N}_{2}$ separation. Their approach utilized low level computational simulations to initially assess the physical characteristics (pore diameters, etc.) of each MOF, and applied increasingly complex and accurate simulation techniques on the MOFs with the most potential. This eventually led to a few high performing MOFs proposed for further experimental analysis. The success of computational screening studies like the examples above is highly dependent on the model being used for adsorption predictions. ${ }^{9,10}$ When the model fails to describe the correct adsorption mechanism, the results can be disastrous. An example of this was highlighted in work by Keskin et al., ${ }^{10}$ which investigated a model for hydrogen adsorption in IRMOF-1 proposed by Yang et al. ${ }^{11}$ The model had been fitted against experimental data up to 1 bar and reproduced the experimental isotherm well within this region. However, Yang et al. ${ }^{11}$ went on to use the model to predict adsorption up to 100 bar, and Keskin et al. ${ }^{10}$ later compared this to experimental values and observed that the simulation uptake of hydrogen was over double the experimental value. The need for robust models is therefore paramount for obtaining reliable results in molecular simulation, even when considering seemingly simple adsorbates such as hydrogen.

One phenomenon observed in some classes of MOFs is the presence of coordinatively unsaturated sites (CUS), also known as open metal sites (OMS). There are various MOFs that develop unsaturated metal centers upon the activation step prior to adsorption. ${ }^{12}$ This occurs when the MOF's metal sites are not fully coordinated to organic linkers but rather to a guest molecule, for instance, the solvent used in synthesis; upon removal of this molecule, the metal is left with a free unsaturated site. These sites have demonstrated selective adsorption for many practical applications, strongly adsorbing gases such as $\mathrm{CO}_{2},{ }^{13} \mathrm{C}_{2} \mathrm{H}_{4},{ }^{14}$ and $\mathrm{H}_{2} \cdot{ }^{15}$ One promising use of this selectivity feature is in the case of olefin/paraffin separations (e.g., ethylene/ethane or propylene/propane), which are generally achieved through costly cryogenic distillation, and for which these CUS-containing MOFs have the potential to be attractive materials for gas separation through alternative techniques such as pressure swing adsorption (PSA). ${ }^{16}$ In the case of olefins, the double bond of the hydrocarbon can strongly coordinate with the metal center of the MOF as explained by the Dewar-ChattDuncanson model. ${ }^{17,18}$ As discussed by Nechaev et al., ${ }^{19}$ this occurs through an electron donation from the olefin's $\pi$-orbital to vacant orbitals of the metal center, with back-donation occurring from the metal's d-orbitals to the antibonding $\pi$ orbital of the adsorbate. ${ }^{20-22}$ The presence of these CUS introduces additional complexity in simulations. Standard force fields were not originally designed to account for these complex orbital interactions, ${ }^{9}$ but rather deal solely with van der Waals forces. In the case of some polar adsorbates, such as water, the inclusion of Coulomb electrostatics within these standard force fields can lead to relatively good agreement between simulation and experiment; ${ }^{23}$ however, this is highly dependent on the selection of point charges. For nonpolar adsorbates such as ethylene, the role of electrostatics has been observed to be insignificant and cannot account for the increased adsorption at the CUS. ${ }^{24}$ This would suggest that the better performance of standard models for polar adsorbates is caused by electrostatics dominating the adsorption mechanism and not by correctly capturing this orbital interaction. ${ }^{25}$ Therefore, a fully transferable model is still required to treat CUS interactions consistently.

A number of approaches have been developed to address this failing, generally achieved through either reparameterization of the existing force fields ${ }^{20}$ or through coupling of GrandCanonical Monte Carlo (GCMC) simulations with complex quantum mechanical calculations. ${ }^{26-28}$ The work by Lamia et al. ${ }^{20}$ focused on the prior method and observed improvement in adsorption isotherm agreement for ethylene by refitting the LJ parameters for the $\mathrm{Cu}-\mathrm{CH}_{2}$ interaction. However, later work by Jorge et al. illustrated that this method did not capture the correct adsorption mechanism and the short-range nature of the orbital interaction. ${ }^{21}$

The latter method of coupling quantum-mechanical calculations with GCMC has led to a successful description of both adsorption isotherms and mechanism through a variety of approaches. Chen et al. ${ }^{26}$ utilized Density Functional Theory (DFT) calculations with a pairwise correction term (DFT/CC) to obtain full energy grids to account for the solid-fluid interactions between methane and HKUST-1 in GCMC simulations. This has the advantage of not having to fit the energy profiles to a function; however, it requires extensive computing power to generate the potential energy map. ${ }^{9}$ Also, it is only currently applicable to spherical adsorbates, which limits it potential transferability to other systems. Dzubak et al. ${ }^{27}$ used DFT calculations to successfully sample $\mathrm{CO}_{2}$ adsorption in both Mg-MOF-74 and MOF-5 (only the former contains CUS). The DFT interaction energy profiles thus created were fitted to a function, and the resulting GCMC simulations obtained good agreement with experimental isotherms. The transferability of this model was also tested by simulating adsorption of $\mathrm{CO}_{2}$ in $\mathrm{Zn-MOF-74}$. Interestingly, only the metal interaction had to be reparameterized in relation to Mg-MOF-74 to obtain good agreement with experiment. As highlighted by Fischer et al., ${ }^{9}$ this transferability suggests that the CUS orbital interaction could potentially be treated independently from the other interactions. It is important to note, however, that the DFT fitting approach of Dzubak el al. led to significantly different parameters for atoms in similar chemical environments (e.g., aromatic carbons) in MOF-74 and MOF-5. This raises questions about whether this DFT approach can be used as a replacement for standard atomic force fields, as a balance must be struck between accuracy and transferability.

The present work expands upon the method of Fischer et al., ${ }^{28}$ which also utilizes DFT energy profiles. Fischer et al. ${ }^{28}$ used DFT to obtain an interaction energy profile between ethylene molecules and HKUST-1 Cu atoms. The major difference of this approach, however, is that the CUS contribution is isolated rather than left grouped with the van der Waals contribution, as in the work of Dzubak et al. ${ }^{27}$ The isolation is achieved by subtracting the contribution of other energy terms determined using classical force fields from the DFT energy profiles. This approach seeks to capitalize on the transferability demonstrated by Dzubak et al. ${ }^{27}$ between $\mathrm{Mg}$ - 
MOF-74 and Zn-MOF-74, which implied that the CUS interaction may be treated independently of other forces. The GCMC simulations then used classical force fields to describe the van der Waals component of the intermolecular forces between the MOF and the adsorbate, while using a specific functional form to capture the CUS interaction. The resulting adsorption isotherms showed very good agreement between simulation and experiment across a variety of temperatures. A major strength of this approach is that the model is able to respond to improvements in the description of either CUS or van der Waals forces independently. Furthermore, as the nonorbital interaction will be captured through classical simulation methods, only one DFT interaction energy profile is required relative to the metal center, describing the CUS contribution. The main advantage, however, is the prospect for the model to be transferable. For example, if the strength of the CUS contribution for the carbon-carbon $\pi$ bond is consistent across olefins, this enables the same force field parameters to be used for various adsorbates. A later paper by Jorge et al. ${ }^{24}$ in fact demonstrated that the same CUS parameters were transferable between ethylene and propylene without the need for any parameter adjustments.

This Article will demonstrate that our CUS model for ethylene is transferable not only to other adsorbates but also to other adsorbent materials with unsaturated $\mathrm{Cu}$ sites. All MOFs studied here contain the copper paddlewheel motif found in HKUST-1 and used in our parameter fitting, but one of the MOFs introduces a different copper building unit. Furthermore, we report some technical improvements in both the DFT and the GCMC procedures. The ultimate aim is to develop a transferable method that correctly captures the CUS behavior in a range of MOFs to enable effective computational screening of CUS-containing MOFs for challenging gas separations. While this Article was being written, we became aware of a very recent study by Kulkarni and Sholl $^{29}$ that shares a similar purpose. Although their approach is conceptually similar to ours, there are some important technical differences that we discuss in detail in section 2.2. Additionally, our focus is on the transferability of the model, so a detailed comparison with experimental pure-component isotherms is central to our goal. Indeed, we show that our approach for describing the CUS interaction leads to very good agreement with experiment, and thus our force field can be used with confidence to make predictions of adsorption in this class of materials.

\section{METHODOLOGY}

This section will outline our procedure to model the interaction of ethylene with the HKUST-1 CUS; however, for a more indepth look into the development of this model, the reader is referred to our previous papers. ${ }^{24,28,30}$ The procedure is comprised of five key stages: (1) quantum-mechanical (QM) calculations to obtain the interaction profile between the adsorbate and the CUS; (2) isolation of the CUS contribution in the DFT profile; (3) fitting the resulting profile to a modified Morse functional; (4) including the new CUS interaction site in GCMC simulation; and (5) validating GCMC adsorption isotherms against experiment. The number of copper paddlewheel MOFs that the new model can be validated against is limited by the number of available experimental isotherms for ethylene adsorption. He et al. ${ }^{31}$ published a number of new ethylene adsorption isotherms for six $\mathrm{Cu}$ containing MOFs: HKUST-1, PCN-16, NOTT-101, MOF-505, UMCM-150, and UTSA-20. Therefore, these MOFs will be the focus of our transferability study. He et al. ${ }^{31}$ also provide methane and ethane adsorption isotherms for these MOFs. As they are both fully saturated hydrocarbons, the CUS interaction will not play an important role in adsorption. ${ }^{9,32}$ Therefore, comparison of simulations with experiment for these adsorbates can be used first to indicate whether the MOF structure and van der Waals interactions are being correctly described in GCMC simulations, before any discrepancies found within ethylene simulations can be attributed to the new CUS model. We have therefore carried out simulations of methane, ethane, and ethylene at both 273 and $296 \mathrm{~K}$ on the six MOFs mentioned above. Initially, new CUS parameters for HKUST-1 were developed to improve on those used in previous work, ${ }^{24}$ and as such the resulting simulated ethylene isotherms were compared to both experiment and isotherms obtained using the previous CUS parameters. Furthermore, additional DFT energy profiles and CUS parameters were obtained for MOF-505 to confirm transferability of the adsorption energies to different MOFs. New energy profiles and CUS parameters were also obtained for the different copper building unit present within the structure of UMCM-150 (see Results and Discussion).

2.1. DFT Calculations. In this work, the DFT calculations use the open source software $\mathrm{CP} 2 \mathrm{~K}^{33,34}$ with periodic boundary conditions, enabling the full MOF structure to be taken into account. CP2K uses a combination of basis sets from Gaussiantype orbitals and a plane-wave basis. ${ }^{33,34}$ The exchangecorrelation functional used was $\mathrm{PBE},{ }^{35}$ as this functional has been used successfully for this model in our previous work. ${ }^{24,28}$ Additionally, it has been shown to capture the correct binding distances for both hydrogen and ethyne on HKUST-1 CUS. $^{30,36}$ PBE does however have limitations regarding treatment of dispersive interactions, ${ }^{37-39}$ which will be discussed and accounted for in the fitting procedure. The basis sets used for all atoms were double- $\zeta$ plus polarization (DZVP) with PBE optimized Goedecker pseudopotentials, ${ }^{34,40,41}$ although TZVP basis sets were also used for benchmark purposes in some cases and found to provide results comparable to those calculated with the more affordable DZVP basis set. The energy cutoff selected was $400 \mathrm{Ry}$, and convergence was checked by using higher values for selected cases, and the calculations used $\Gamma$ point sampling. Furthermore, spin polarization was accounted for in all DFT calculations. Initially, both the ethylene and the HKUST-1 structures were optimized independently and later used as reference energies to obtain the ethylene-MOF interaction energy from

$$
U_{\text {interaction }}(r)=U_{\text {complex }}(r)-U_{\text {HKUST-1 }}(r)-U_{\text {ethylene }}(r)
$$

This differs from our previous work, ${ }^{24}$ in which the original HKUST-1 CUS parameters were obtained using energy profiles from all-electron DFT cluster calculations ran with the $\mathrm{DMol}^{3}$ $\operatorname{code}^{42,43}$ contained within the "Material Studio" package. ${ }^{44}$ In cluster calculations, only a section of the MOF structure is considered, unlike periodic calculations that repeat a specific unit cell in three dimensions leading to a full (infinite) MOF structure representation. Small cluster models behave well in cases where the nature of the interaction is very localized but can lead to inaccurate representations of molecular systems when long-range effects are important. ${ }^{37}$ As such, the decision was made to switch to periodic DFT calculations to remove this potential source of error (and, in the process, validate the accuracy of our previous cluster calculations). Furthermore, and above all, using periodic DFT calculations improves consistency 
with later steps in the fitting procedure, which are also based on periodic boundary conditions (see Parameter Fitting).

The DFT optimization protocol differed only slightly from the work of Jorge et $\mathrm{al}^{24}$ due to the introduction of periodic calculations; it should be noted however that both present and previous $^{24}$ DFT calculations use two adsorbing ethylene molecules on either side of a copper dimer to limit distortion of the copper building unit. First, after the initial minimum energy geometry optimization of the ethylene-MOF complex, the internal degrees of freedom of the adsorbate were kept entirely fixed, while the adsorption energy profile along the $\mathrm{Cu}-\mathrm{Cu}$ vector of the paddlewheel was computed. This was required as the adsorbate is now in a $3 \mathrm{D}$ cage and could move away from the relevant $\mathrm{Cu}-\mathrm{Cu}$ vector at larger distances from the metal, where the CUS interaction becomes weaker and van der Waals forces dominate. This has been extensively studied previously by our group with cluster calculations, and the effect of fixing the ethylene molecule internal geometry along the adsorption profile is negligible. Conversely, as the DFT calculations were now periodic, there was the opportunity to allow the entire framework to relax upon optimization with ethylene; this was not possible in our previous cluster calculations because it would lead to unphysical distortion of the MOF cluster. Table 1 compares the optimized energies and

\section{Table 1. DFT Calculations with Different Geometry} Constraints

\begin{tabular}{clcc}
$\begin{array}{c}\text { DFT } \\
\text { calculation }\end{array}$ & \multicolumn{1}{c}{ optimization } & $\begin{array}{c}\text { int. energy } \\
(\mathrm{kJ} / \mathrm{mol})\end{array}$ & $\begin{array}{c}\text { binding distance } \\
(\AA)\end{array}$ \\
\hline cluster $^{24}$ & relaxed copper & -23.3 & 2.60 \\
periodic & fixed framework & -21.5 & 2.74 \\
periodic & relaxed copper & -23.3 & 2.63 \\
periodic & relaxed framework & -23.7 & 2.61 \\
\hline
\end{tabular}

binding distances ( $\mathrm{Cu}$ to the center of the ethylene double bond) obtained in a fully fixed HKUST-1 framework, a fully relaxed framework, and a partially relaxed framework, in which only the $\mathrm{Cu}$ atoms were free to move. Using a fixed framework led to a slightly less favorable binding energy, and correspondingly larger binding distance, for ethylene, as observed previously. ${ }^{28}$ However, the difference in interaction energy between a partially restricted optimization $(\mathrm{Cu}$ free to relax) and a nonrestricted framework was not significant, and therefore the former approach was chosen to minimize computing time. We note that the interaction energies shown in Table 1 do not properly account for dispersion interactions. In Figure S1, we plot the full DFT profile obtained with the chosen protocol, as well as the resulting profile when LJ interactions are added.

2.2. Parameter Fitting. Once the interaction energy profiles between the adsorbate and the metal site of a MOF were calculated using DFT, the CUS contribution must be isolated before it can be fit to a given functional form, and this requires assumptions about the DFT energies. The first assumption is that electrostatics do not play a significant role in ethylene adsorption on MOF frameworks. Jorge et al. ${ }^{24}$ simulated adsorption isotherms for ethane and ethylene in HKUST-1 both explicitly including and ignoring electrostatic interactions, and also without including a CUS specific interaction. The difference between the isotherms was negligible, indicating that the above assumption is reasonable and that the electrostatic contribution to the energy profile can be disregarded. The second major assumption is that the PBE exchange-correlation functional does not capture any dispersive van der Waals interactions near the $\mathrm{Cu}$ site; PBE often predicts very low or negligible binding in van der Waals complexes. ${ }^{37-39}$ This then implies that the energy of interaction obtained in DFT arises solely from the $\mathrm{Cu}-\pi$ interaction and the repulsive contribution: ${ }^{28}$

$$
U_{\mathrm{Cu}-\pi}(r)=U_{\mathrm{DFT}}(r)-U_{\text {repulsive }}(r)
$$

To isolate the $\mathrm{Cu}-\pi$ interaction, the repulsive contribution must thus be determined. This was achieved using in-house software to calculate the Lennard-Jones (LJ) potential energy profile along the $\mathrm{Cu}-\mathrm{Cu}$ vector in a fully periodic structure, based on the models that will be used later in GCMC simulations. The Weeks-Chandler-Andersen (WCA) approximation $^{45,46}$ was then applied to isolate the repulsive contribution in the LJ potential; see Figure S2.

The now isolated $\mathrm{Cu}-\pi$ profile represents the attractive interaction energy between the olefin double bond and the copper atom due only to the CUS, and will need to be fit to a function for use in GCMC simulations by adding an additional interaction site located at the center of the ethylene double bond. The functional form selected was a five-parameter modified Morse potential, ${ }^{28}$ shown in eq 3 . This includes a power law term enabling the short-range nature of the CUS interaction to be fully captured.

$$
\begin{gathered}
U_{\text {function }}(r)=D_{\mathrm{o}} \cdot\left[\exp \left(\alpha\left(1-\frac{r}{R_{\mathrm{o}}}\right)\right)\right. \\
\left.-2 \cdot \exp \left(\frac{\alpha}{2}\left(1-\frac{r}{R_{\mathrm{o}}}\right)\right)\right]-\left(\frac{A}{r}\right)^{B}
\end{gathered}
$$

In this equation, $R_{\mathrm{o}}$ corresponds to the distance of the minimum in the Morse potential, while $D_{\mathrm{o}}$ represents the depth of this minimum, akin to the role of $\sigma$ and $\varepsilon$, respectively, in the LJ potential. The $\alpha$ constant reflects the flexibility of the curve, while the final two parameters $A$ and $B$ are purely empirical terms.

In our previous work, ${ }^{21}$ eq 3 was fitted to the energy profile over a range of binding distances between 2 and $5 \AA$. A high cutoff at larger separation distances was then introduced into the GCMC simulations for this $\mathrm{Cu}-\pi$ interaction to avoid an unrealistic enhancement of adsorption energies away from the $\mathrm{Cu}$ atom. ${ }^{24}$ This would contradict the short-range and highly directional nature of the $\mathrm{Cu}-\pi$ interaction, and may violate the requirement that the unsaturated copper can only form a coordination bond with one adsorbate molecule at a time. This was complemented by a smooth shift cutoff region to avoid a discontinuity in the potential; as an adsorbate molecule's binding distance enters the shift cutoff region, its interaction energy will gradually tend toward zero as the binding distance approaches the high cutoff value. The final cutoff scheme has the following form:

$$
U_{\mathrm{Cu}-\pi}(r)=\left\{\begin{array}{cc}
U_{\text {function }}(r) & \text { shiftcut } \geq r \\
U_{\text {function }}(r) \cdot \frac{(\text { hicut }-r)}{(\text { hicut }- \text { shiftcut })} & \text { shiftcut }<r<\text { hicut } \\
0 & r \geq \text { hicut }
\end{array}\right.
$$

Previously, the high cutoff for this $\mathrm{Cu}-\pi$ interaction was set at $5 \AA$ with the shift cutoff set at $4 \AA^{24}$. We have investigated the cutoff scheme in more depth, and although the previous 
scheme greatly improved upon the issue of unrealistic binding energies, it did not fully solve the problem. This was tested by carrying out GCMC simulations on HKUST-1 preloaded with one ethylene molecule adsorbed at each unsaturated $\mathrm{Cu}$ site, both with and without the CUS-specific interaction term. As the copper sites are preloaded, the CUS interaction should have no effect on adsorption, and thus the two isotherms should be identical. Any differences can thus be ascribed to an artificial enhancement of the CUS interaction energy away from the metal site (for more details, see the Supporting Information). This investigation identified a high cutoff of $4 \AA$ and a shift cutoff of $3.5 \AA$ as optimal (Figures S3 and S4). Because of this improved cutoff scheme, the parameter fitting procedure was only carried out between binding distances of 2 and $4 \AA$. Finally, a low cutoff for the CUS interaction was also introduced to avoid mathematical artifacts in close vicinity to the open metal site. For example, in the case of HKUST-1, we observed a small number of configurations at binding distances between 1 and $1.4 \AA$ in which the negative CUS term was higher in magnitude than the positive repulsive energy from the LJ potential, which created an unrealistic adsorption site very close to the copper atom. This artifact was eliminated by shifting the CUS interaction energy to zero below a low-cut value of $1.8 \AA$. Although these changes to the cutoff scheme had a very small effect on the ethylene adsorption isotherms in HKUST-1 (Figure S5), there is no guarantee that the effect will not be important in other MOFs or with other adsorbates. As such, we have implemented the new cutoff scheme in our calculations and recommend it for future work, as, although the effect of the low cutoff on the isotherm may be minor, its description of the binding mechanism will be more physically reasonable.

It is worth mentioning at this stage that our parameter fitting procedure is quite different from that proposed recently by Kulkarni and Sholl. ${ }^{29}$ Those authors chose to fit their DFT energies to a pairwise Morse potential between the $\mathrm{Cu}$ atom and each of the $\mathrm{CH}_{2}$ sites of ethylene, whereas we have added a new CUS-specific interaction site at the center of the double bond. Their original scheme led to artificially high interaction energies for configurations in which the olefin was highly tilted with respect to the $\mathrm{Cu}-\mathrm{Cu}$ vector, which prompted the authors to introduce a three-body orientationally dependent term to their potential. Furthermore, because their CUS interactions are truncated only at $13 \AA$, it is possible that their model is artificially enhancing adsorption away from the $\mathrm{Cu}$ atom (we will return to this point later in this Article). On the contrary, our fitting scheme ensures that the short-range and directional nature of the $\mathrm{Cu}-\pi$ interaction is correctly captured.

2.3. GCMC Simulations. All adsorption isotherms were obtained through GCMC simulations using the open source code Music. ${ }^{47}$ The code was adapted to include the modified Morse potential function, enabling CUS interactions to be introduced. GCMC simulations keep the system's chemical potential $(\mu)$, volume $(V)$, and temperature $(T)$ all fixed, while allowing the number of molecules $(N)$ to vary. Before full GCMC adsorption simulations were run, potential maps (PMAPs) that tabulate the interaction energy between a given adsorbate site and the MOF on a fine grid were produced. The grid spacing selected for these maps was $0.15 \AA$. Using these maps reduces the simulation time, as the interaction energy between the adsorbate and the MOF can simply be interpolated rather than calculated on the fly for each simulation step. For simulations of methane and ethane, cavity bias ${ }^{48}$ based on the LJ potential maps was used for insertion and deletion trials. This bias enables steps not to be wasted on positions that are irrelevant to adsorption, such as those that overlap with framework atoms. In the case of ethylene, however, insertion and deletion were done randomly (i.e., without bias) as two map types were present (CUS and LJ), and using a bias based on only one map type could possibly negatively deviate the simulations away from sites that would become favorable when accounting for both intermolecular forces. In the future, we intend to further modify the simulation code to allow for biased insertions and deletions using a combination of PMAPs. In addition to insertion and deletion trials, molecules were allowed to rotate and translate (or only translate, in the case of methane), using optimized maximum displacements. All MC trials were equally weighted and occurred once per simulation step. A minimum of 100000000 steps were used, but this was increased to 500000000 for methane simulations to reduce statistical error due to the smaller amounts adsorbed. The first $50 \%$ of steps were ignored to ensure equilibration, and the remaining steps were split into 20 equal blocks for error analysis. Error bars were always smaller than the symbols used in the Results and Discussion and are therefore not shown. Pressure values were converted to fugacities for input into the simulation code using the Peng-Robinson equation of state. ${ }^{49}$ The final absolute adsorbed amounts from the simulations were converted to excess, for comparison with experiment, using the Myers and Monson method. ${ }^{50}$

The DREIDING force field ${ }^{51}$ was used to describe most framework atoms as it has previously been successful in describing adsorption in MOFs. ${ }^{52-54}$ The exception is the copper atom, which is not present in DREIDING; as such, the Universal force field (UFF) ${ }^{55}$ was selected to describe the metal. For the adsorbates, a united atom approach was used for each $\mathrm{CH}_{x}$ group, with parameters taken from the TraPPE force field. ${ }^{56,57}$ Both the framework and the adsorbates were kept fully rigid during simulations. All cross-species LJ parameters were determined using the standard Lorentz-Berthelot combining rules, and an interaction cutoff distance of $13 \AA$ was applied. The exception was the interaction between the new site, located at the center of the ethylene double bond, and the unsaturated $\mathrm{Cu}$ atom of the MOF, for which the cutoff scheme described in the previous section was applied. The values of the cutoff distances are provided in Table 2 for

Table 2. CUS Interaction Cutoff Scheme

$\begin{array}{ll}\text { low cutoff } & 1.8 \AA \\ \text { shift cutoff } & 3.5 \AA \\ \text { high cutoff } & 4.0 \AA\end{array}$

completeness, while Table 3 reports the CUS parameters found through fitting the DFT energy profiles obtained on three different MOFs to eq 3, as well as the corresponding parameters obtained in our previous work. ${ }^{24}$

2.4. Structural Analysis. As various MOFs will be used to check the transferability of our CUS model, it is important to understand the underlying MOF structures being simulated. The open source software Poreblazer ${ }^{58}$ was therefore used as a structure analysis tool for all MOF structures studied in this work. Among other metrics, Poreblazer calculates the framework density, pore volume, accessible surface area, and geometric pore size distribution, enabling a better under- 
Table 3. CUS Parameters Obtained from DFT Fitting to Equation 3

\begin{tabular}{|c|c|c|c|c|c|c|c|}
\hline MOF & $\mathrm{Cu}$ complex & produced & $R_{\mathrm{o}}(\AA)$ & $D_{\mathrm{o}}(\mathrm{kJ} / \mathrm{mol})$ & $\alpha$ & $A$ & $B$ \\
\hline HKUST-1 & dimer & Jorge et al. ${ }^{24}$ & 3.1870 & 9.9600 & 7.9200 & 3.867 & 9.2400 \\
\hline HKUST-1 & dimer & this work & 3.1030 & 10.6324 & 8.0945 & 3.825 & 9.2812 \\
\hline MOF-505 & dimer & this work & 3.3484 & 9.8712 & 6.8640 & 4.007 & 8.5900 \\
\hline UMCM-150 & trimer & this work & 4.3090 & 8.0858 & 5.9320 & 4.337 & 8.7583 \\
\hline
\end{tabular}

standing of the number of cage types within each MOF. Importantly, it also provides an estimate of the pore limiting diameter for each MOF, which is the narrowest constriction found in any path that permits a guest molecule to diffuse through the MOF structure. ${ }^{58}$ It thus provides some important insight into the pore network topology and may help identify existing diffusion limitations. However, it does not always correspond to the smallest window size in a given MOF; in cases where pores are isolated from the rest of the network by small windows, they will not be part of the diffusive path of the adsorbates. Analysis of the entire pore network connectivity is used to identify possible regions of the pore space that may be inaccessible for adsorption. A full breakdown of the Poreblazer analysis on all of the studied MOFs can be found in Table S1. Also, the following files are available free of charge on the University of Strathclyde's data repository: input files and source code for all GCMC simulations (DOI: 10.15129/ 8e3b13eb-7625-4eaa-a658-1f6be4e2478a), and input files for all DFT calculations (DOI: 10.15129/16b6418d-d728-4636-bb289d1d0fe42e63).

\section{RESULTS AND DISCUSSION}

In this section, we will present the results of transferring the CUS parameters obtained on HKUST-1 to various other $\mathrm{Cu}$ based MOFs. In previous work, we have demonstrated that our approach is able to correctly describe the adsorption mechanism of olefins on HKUST- $1,{ }^{28}$ and that the parameters are transferable between different adsorbate molecules of the same type (e.g., ethylene to propylene) on that same MOF material. ${ }^{24}$ Nevertheless, we first revisit ethylene adsorption in HKUST-1 to compare our model predictions to the experimental data set of $\mathrm{He}$ et al., ${ }^{31}$ which was not considered in our previous work, and to validate the changes introduced in the DFT calculations (periodic instead of cluster) and fitting procedure (slightly improved cutoff scheme). For that purpose, we compare isotherms obtained using our new approach to those using the original parameters of Jorge et al. ${ }^{24}$ Subsequently, adsorption isotherms using parameters obtained from periodic DFT calculations on HKUST-1 will be calculated for the remaining MOFs, PCN-16, NOTT-101, MOF-505 (NOTT-100), UMCM-150, and UTSA-20, and compared against experimental data. ${ }^{31}$ It should be noted that all experimental isotherms were scaled up using the ratio between the theoretical pore volume of an ideal crystal and the experimental pore volume reported by $\mathrm{He}$ et al., ${ }^{31}$ both determined from analysis of saturated nitrogen adsorption at 77 $\mathrm{K}$. This procedure takes into account in an approximate way the observed reduced adsorption capacity in real MOF samples, due to potential imperfections such as collapsed pores, nonporous byproducts, and solvent molecules trapped inside the pores of the MOF during synthesis. ${ }^{24}$

3.1. HKUST-1. HKUST-1 $1^{59}$ is one of the most widely studied MOFs. ${ }^{60}$ It has the composition $\mathrm{Cu}_{3}(\mathrm{BTC})_{2}$ and is made up of two copper ions in a paddle wheel structure with BTC linkers, benzene-1,3,5-tricarboxylate (Figure 1). ${ }^{61}$ The

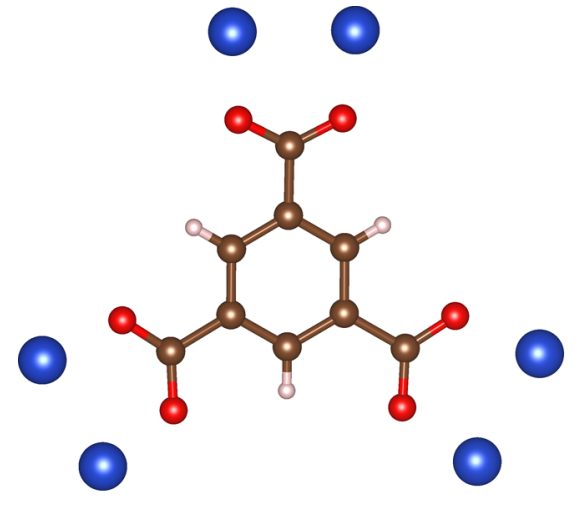

(a) Linker

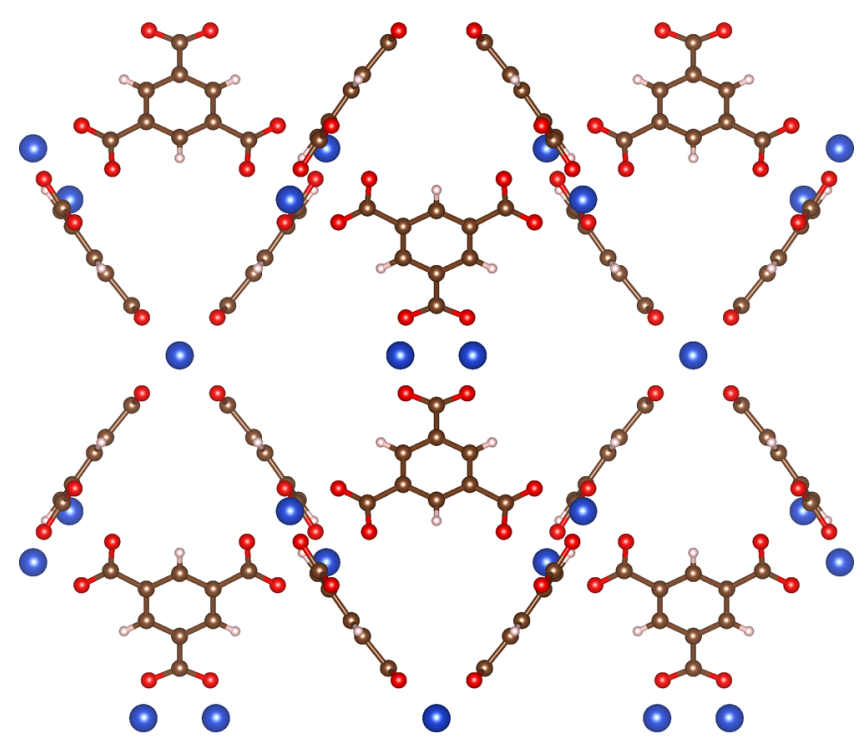

(b) Unit Cell

Figure 1. Diagram showing the linker (a) and the unit cell topology (b) of HKUST-1. Color code for atoms is blue, copper; red, oxygen; brown, carbon; and white, hydrogen.

Table 4. Dimensions of Adsorbates

\begin{tabular}{llll} 
molecule & $x(\AA)$ & $y(\AA)$ & $z(\AA)$ \\
methane $^{64}$ & 3.829 & 4.101 & 3.942 \\
ethane $^{64}$ & 3.809 & 4.079 & 4.871 \\
\hline
\end{tabular}

overall structure is shown to be cubic with a pto topology, ${ }^{30}$ which arises from the tritopic linker. The secondary building unit (SBU) is made up of the two copper atoms bridged by four carboxylate functionalities from the BTC linker. ${ }^{61} \mathrm{Cu}_{3}(\mathrm{BTC})_{2}$ has three pore types, all roughly spherical: one small tetrahedral pore of $\sim 5 \AA$ and two larger octahedral pores of $\sim 11$ and 12 $\AA^{62,63}$ The CUS sites within HKUST-1 face the interior of the largest pore, $12 \AA$. Poreblazer ${ }^{58}$ identified a pore limiting 


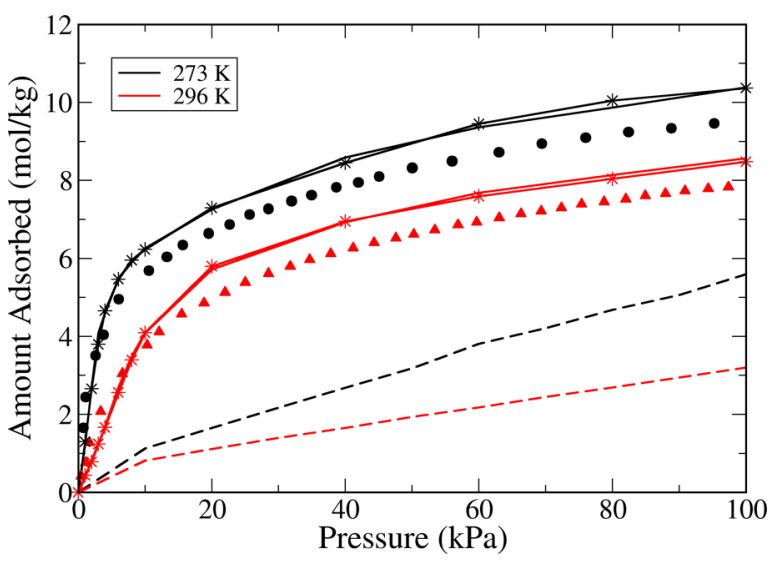

Figure 2. Comparison between simulated (lines) and experimental ${ }^{31}$ (symbols) ethylene adsorption in HKUST-1: solid line, periodic DFT model; starred line, cluster DFT model; dashed line, standard LJ model (without CUS interaction).

diameter of $6.4 \AA$ (see Table S1). This can be compared to the dimensions of the adsorbates, shown in Table 4 (only data for methane and ethane were found, ${ }^{64}$ but we expect the dimensions of ethylene to be quite similar to those of ethane). It can be seen that the kinetic diameters of all three adsorbates are significantly smaller than the pore limiting diameter, and thus no significant diffusion limitations are expected.

We use ethylene adsorption on HKUST-1 to validate the new parameters for the $\mathrm{Cu}-\pi$ interaction obtained in this work, as well as the changes implemented in the parameter fitting procedure. As illustrated in Figure 2, the CUS parameters derived from periodic DFT calculations lead to predicted isotherms that are practically indistinguishable from those obtained with parameters derived from our previous cluster calculations. ${ }^{24}$ Furthermore, both sets of predicted isotherms agree very well with the experimental data of $\mathrm{He}$ et al. ${ }^{31}$ at both reported temperatures. This is in marked contrast to the results obtained with the standard DREIDING potential (i.e., without including the specific $\mathrm{Cu}-\pi$ interaction), which dramatically underestimate adsorption. It should be noted that the experimental pressure range for this data set is much lower than for the data used in our previous work, which went up to 7 bar. ${ }^{24}$ This makes the validation even more stringent as it focuses on the region in which solid-fluid interactions are dominant, and therefore where the effect of the CUS interaction will be most important.

The good agreement obtained with both DFT-based approaches confirms that the MOF cluster we employed previously $^{28}$ was large enough to accurately capture the local

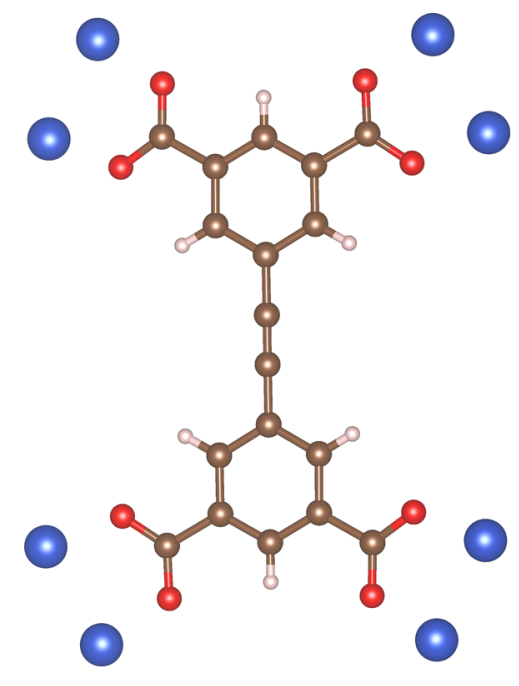

(a) Linker

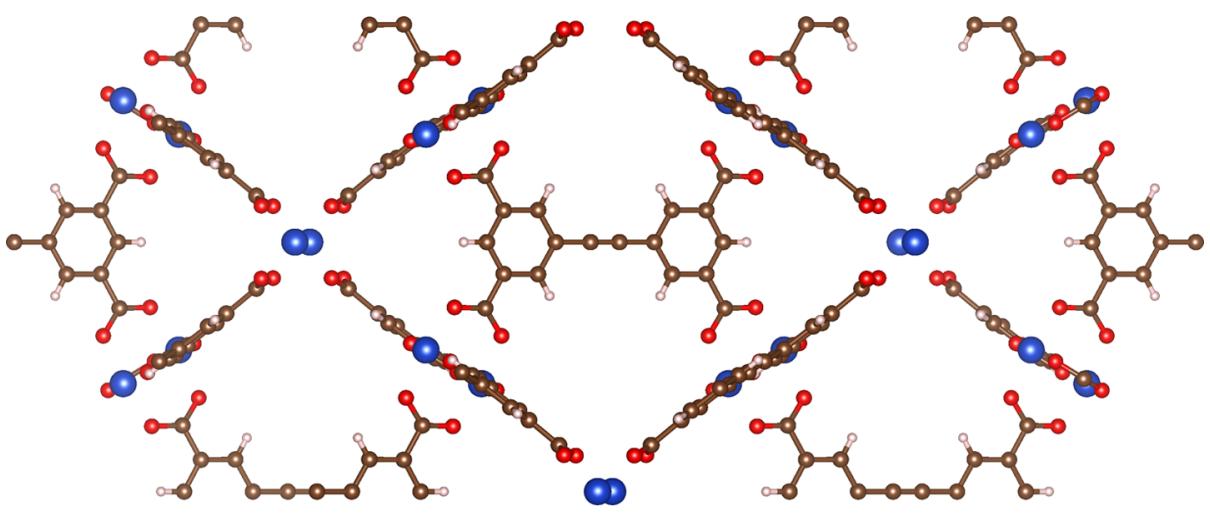

(b) Topology

Figure 3. Diagram showing the linker (a) and the unit cell topology (b) of PCN-16. Color code is the same as in Figure 1. 

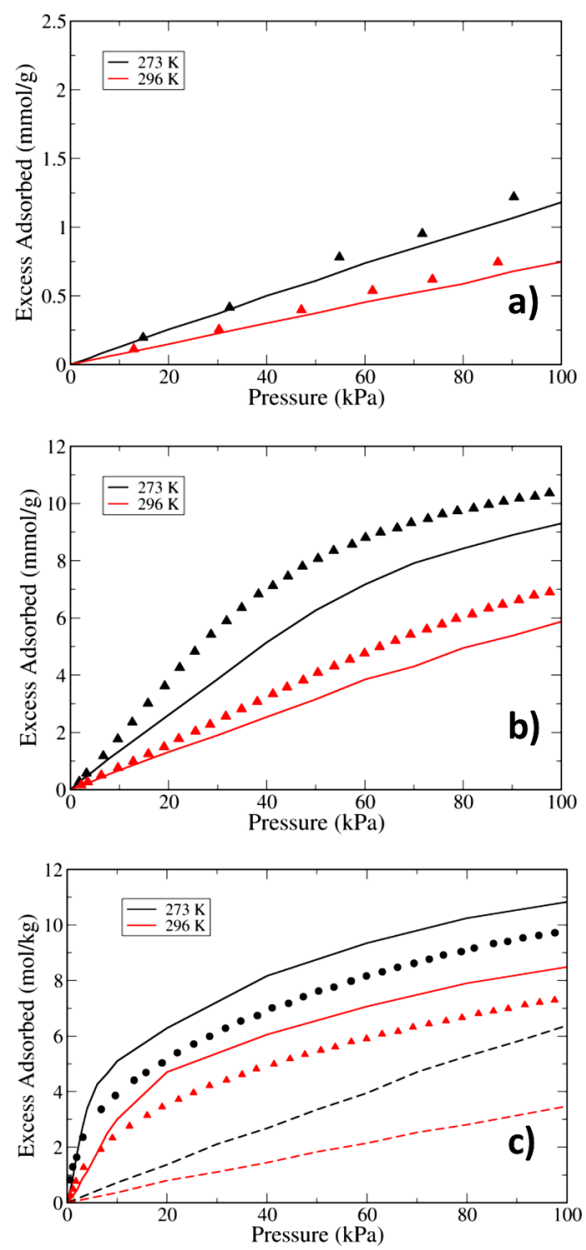

Figure 4. Simulated (lines) and experimental ${ }^{31}$ (points) adsorption isotherms in PCN-16 for (a) methane, (b) ethane, and (c) ethylene. For ethylene, the solid line shows results of the periodic DFT model, and the dashed line is for the standard LJ model (without CUS interaction).

Table 5. RMSD between Simulated and Experimental Adsorption Isotherms

\begin{tabular}{|c|c|c|c|}
\hline MOF & $\begin{array}{l}\text { methane } \\
(\mathrm{mmol} / \mathrm{g})\end{array}$ & $\begin{array}{c}\text { ethane } \\
(\mathrm{mmol} / \mathrm{g})\end{array}$ & $\begin{array}{l}\text { ethylene } \\
(\mathrm{mmol} / \mathrm{g})\end{array}$ \\
\hline PCN-16 & 0.093 & 1.112 & 1.083 \\
\hline NOTT-101 & 0.029 & 0.418 & 1.170 \\
\hline MOF-505 & 0.054 & 1.398 & 2.254 \\
\hline MOF-505 (scaled $)^{a}$ & & 0.160 & 0.908 \\
\hline UMCM-150 $\left(\mathrm{Cu}_{2}\right)^{b}$ & 0.062 & 0.504 & 1.325 \\
\hline $\begin{array}{l}\mathrm{UMCM}-150 \\
\quad\left(\mathrm{Cu}_{2} / \mathrm{Cu}_{3}\right)^{c}\end{array}$ & & & 0.997 \\
\hline UTSA-20 & 0.598 & 1.372 & 1.670 \\
\hline UTSA-20 (blocked) $^{d}$ & 0.070 & 0.562 & 0.433 \\
\hline
\end{tabular}

${ }^{a}$ Simulated isotherm scaled by 0.8 accounts for incomplete equilibration of experiment. ${ }^{b}$ All CUS assumed to behave equivalently. ${ }^{c}$ Separate CUS parameters used for trimer SBU. ${ }^{d}$ Small cages blocked.

interactions in the vicinity of the CUS, and that the small changes we introduced to the fitting procedure do not have a significant impact on adsorption predictions for this particular system. Nevertheless, the decision was made from this point forward to use periodic DFT calculations, because as previously mentioned this will improve consistency of the fitting process and eliminate potential inaccuracies due to the limited size of cluster models. In particular, it will likely improve the accuracy of modeling adsorbates where electrostatics play an important role, as these interactions are typically long-ranged in nature. Furthermore, using periodic calculations obviates the need of constructing different cluster models for MOFs that contain more than one inorganic SBU, such as UMCM-150.

3.2. PCN-16. PCN-16 $6^{65}$ has the composition $\mathrm{Cu}_{2}$ (ethynediyl-bis(benzenedicarboxylate)). Similar to HKUST-1, it also contains a copper paddle wheel complex, and the SBU is again made up of the two copper atoms bridged by four carboxylate functionalities. However, the organic linker connects to four copper paddle wheels (Figure 3a), leading to a trigonal structure. The topology of PCN-16 is often described as being of the nbo type; ${ }^{66}$ however, as discussed in more detail by $\mathrm{Li}$ et al., ${ }^{67}$ a description using the fof net gives a more complete representation of the topological features. The same applies to the isoreticular systems MOF-505 and NOTT-101. Additionally, the much longer organic linker, shown in Figure $3 \mathrm{a}$, than in the case of HKUST-1 leads to a unit cell that is elongated in one direction (Figure $3 \mathrm{~b}$ ). This creates two cage types, one octahedral cage that is roughly spherical and has a diameter of $\sim 7 \AA$ and a larger elliptical cage. ${ }^{68}$ These cages are interconnected by two types of windows that lead to a channellike pore structure with a pore limiting diameter of $6.32 \AA$ as identified by Poreblazer (see Table S1). This indicates that the pore network is fully accessible to all three gases. The CUS sites present in this MOF face into the smaller octahedral cage.

As illustrated in Figure 4, experimental isotherms of both methane and ethane are captured relatively well by the standard LJ model across the full pressure range and at two different temperatures, although there is a slight underestimation present in ethane simulations. These simulations did not account for any electrostatics or CUS-specific interaction, suggesting that the adsorption mechanism is mainly dominated by van der Waals forces for those two adsorbates. However, the standard LJ model fails completely at describing ethylene adsorption, especially at the low pressure region (Figure 4c). This failure in the Henry's law region suggests that the solid-fluid interaction is being greatly underestimated, as was already highlighted in our previous work. ${ }^{9}$ In contrast to the linear behavior of the simulations with the LJ model, the experimental isotherm has a steep initial uptake due to the strength of the CUS, and as they begin to fill the isotherm levels off. The new model captures this behavior of the ethylene isotherm very well, especially in the low pressure region.

The good agreement between experiment and simulation for ethylene in PCN-16 using the CUS parameters determined from DFT calculations on HKUST-1 suggests that these parameters are indeed transferable to different $\mathrm{Cu}$-containing MOFs. Nevertheless, a slight overestimation across the entire simulated isotherm is observed. To better quantify the degree of agreement, we have computed the root-mean-squared deviation (RMSD) between simulated and experimental isotherms, calculated at the experimental pressure points (details in the Supporting Information). This is shown in Table 5 for all adsorbates and all MOFs studied in this Article. The systematic overestimation observed for ethylene in Figure $4 \mathrm{c}$ is indeed reflected in a somewhat larger RMSD for that adsorbate, as compared to methane and ethane. However, given that matching the adsorption isotherms at relatively low pressures and temperatures is a rather stringent test of our model, we believe the agreement is satisfactory. 


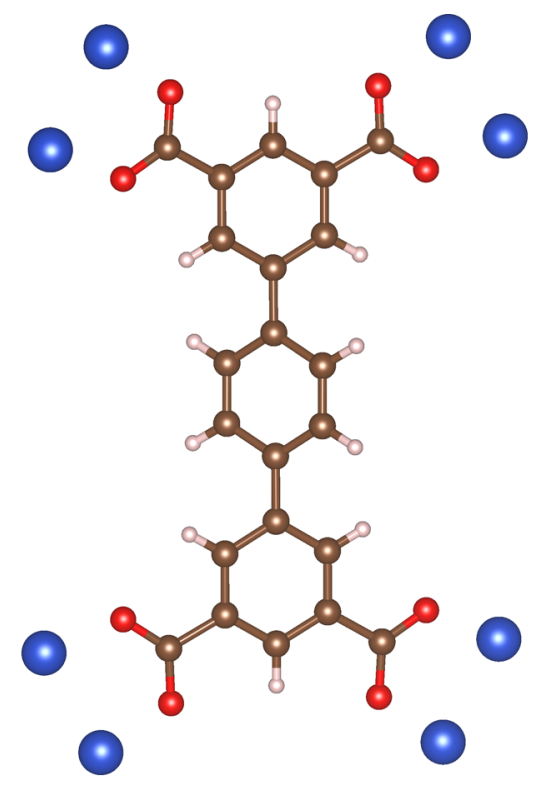

(a) Linker

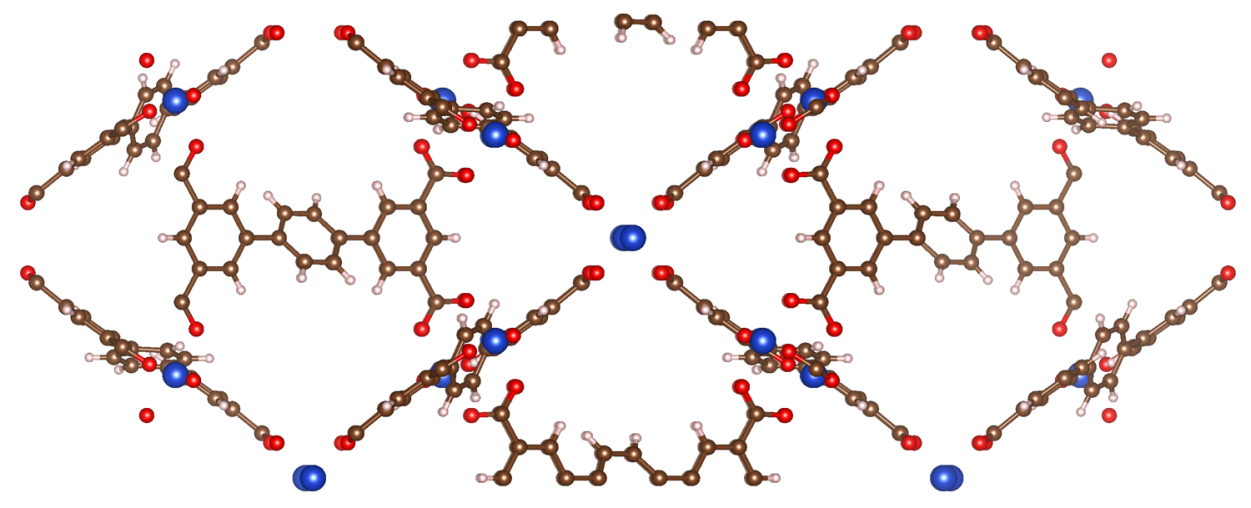

(b) Topology

Figure 5. Diagram showing the linker (a) and the unit cell topology (b) of NOTT-101. Color code is the same as in Figure 1.

3.3. NOTT-101. NOTT-101, ${ }^{69}$ shown in Figure 5b, is very similar to PCN-16, retaining the $f \circ f^{67}$ topology with two pore types interconnected by two triangular windows on one axis. ${ }^{69}$ However, the linker is slightly longer due to the central ethynyl group, found in the PCN-16 linker, being replaced with an aromatic ring connecting the benzene carboxylate moieties, as shown in Figure 5a. In the case of the NOTT family, the connecting windows have been observed to increase in size with increasing linker length. ${ }^{69}$ Poreblazer identified a pore limiting diameter of $5.6 \AA$, which due to the channel nature of the MOF can be attributed to one of these triangular windows. Comparing this value to the dimensions of the adsorbates (Table 4) again suggests the absence of diffusional limitations for the three gases in this MOF.

In the case of NOTT-101, simulations using the DREIDING/TraPPE models predict adsorption of methane and ethane exceptionally well (Figure $6 \mathrm{a} / \mathrm{b}$ ), further confirming the suitability of this model for saturated hydrocarbons. A trend similar to that of PCN-16 can also be observed for ethylene adsorption (Figure 6c). The standard model again fails to identify the CUS as the preferential site and strongly underpredicts adsorption across the whole isotherm. The new transferable model greatly improves predictions and captures the correct shape of the isotherm. Again, a slight overestimation is observed, of magnitude similar to that of PCN-16 (see Table 5 ), suggesting that it may be a systematic issue. However, this was also observed in HKUST-1, albeit to a lesser extent (see Figure 2), implying that this is not a problem caused by transferring the CUS parameters from one MOF to another. We will return to this point later in this Article.

3.4. MOF-505. MOF- $505,{ }^{70}$ also called NOTT-100, is from the same family as NOTT-101 and therefore retains the same fof ${ }^{67}$ topology and general pore structure, which can be observed through comparison of MOF-505 in Figure 7 and NOTT-101 in Figure 5. The main difference from the two previous MOFs is the shorter organic linker, as shown in Figure 7b. As mentioned, the linker length has been shown to affect the window size of this MOF type, in this case creating smaller windows. $^{69}$ This was confirmed using Poreblazer, which reported a smaller limiting diameter of $4.53 \AA$ for MOF-505, as compared to $5.60 \AA$ for NOTT-101. This is now of the same order as some of the adsorbate dimensions (see Table 4), which may anticipate some diffusion limitations.

It can be seen in Figure $8 \mathrm{a}$ that the simulations again accurately capture experimental methane adsorption (the RMSD is similar to that in the previously studied MOFs; see 

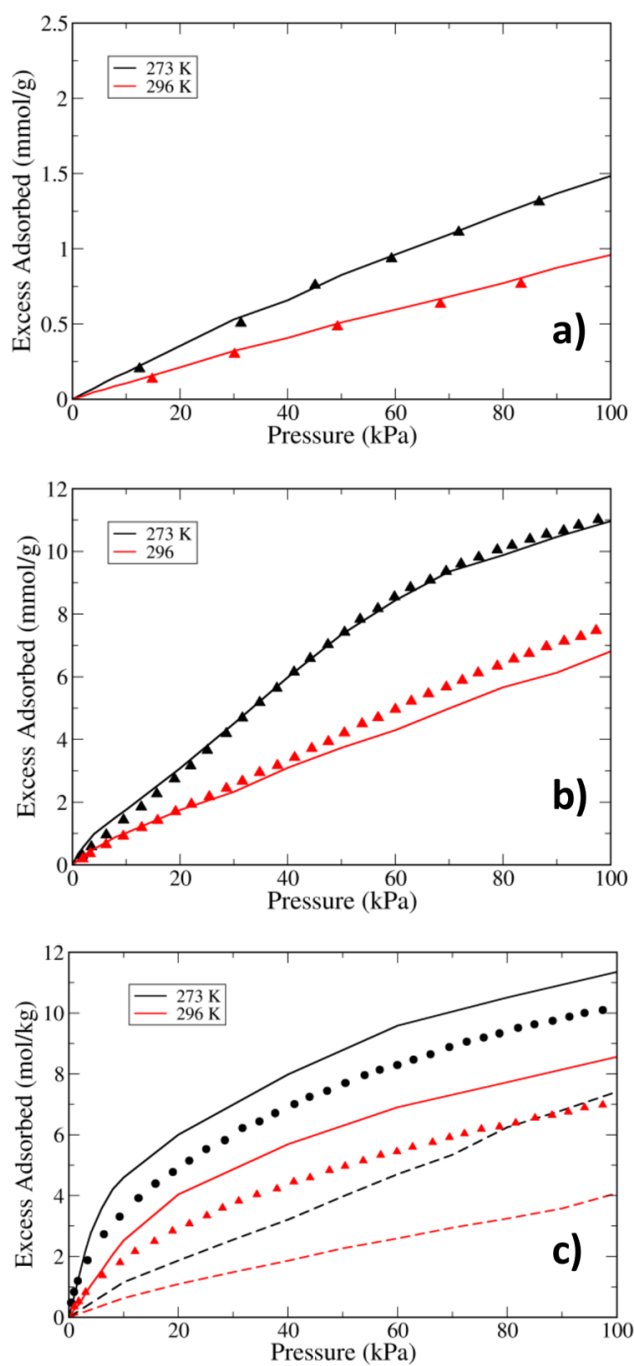

Figure 6. Simulated (lines) and experimental ${ }^{31}$ (points) adsorption isotherms in NOTT-101 for (a) methane, (b) ethane, and (c) ethylene. For ethylene, the solid line shows results of the periodic DFT model, and the dashed line is for the standard LJ model (without CUS interaction).

Table 5). In contrast, simulated ethane adsorption is now systematically overestimated in comparison with experiment (Figure $8 \mathrm{~b}$ ). This is somewhat surprising as both methane and ethane should be attracted to similar adsorption sites, both being fully saturated small hydrocarbons. Figure $8 \mathrm{c}$ shows a similar overestimation of ethylene adsorption from simulations using the new CUS model. This overestimation is much greater than observed for the two previous MOFs, with an RMSD that is about twice that of PCN-16 and NOTT-101 (Table 5). It is also worth noting that the seemingly good quantitative agreement between ethylene simulations using DREIDING and experiment for this MOF results from fortuitous error cancellation, and belies the intrinsic shortcomings of the standard LJ model. Indeed, closer examination of Figure 8c shows that the predicted shape of the isotherm is qualitatively different from experiment, with low pressure adsorption being underestimated, followed by overestimation at higher pressures. Once more, this is due to the inability of the standard approach to describe adsorption at the CUS.

One possible explanation for the systematic overestimation observed in the case of ethylene adsorption is that the CUS interaction parameters transferred from HKUST-1 lead to stronger adsorption energies than observed in MOF-505. This was tested by fitting new CUS parameters from periodic DFT calculations on MOF-505 (Table 3). As the interaction strength at the minimum of the DFT profile was found to be $-22.6 \mathrm{~kJ} /$ $\mathrm{mol}$, in comparison to $-23.3 \mathrm{~kJ} / \mathrm{mol}$ for HKUST-1, this is unlikely to be the cause of the observed overestimation. This was confirmed by running GCMC simulations using the new MOF-505 parameters, shown in Figure 9. These parameters lead to almost identical isotherms as compared to those obtained with the original HKUST-1 CUS parameters, confirming that the CUS interaction for the copper paddlewheels is generally consistent across different MOFs. This also confirms that the overestimation, which is also observed for ethane, is not a consequence of our assumption of parameter transferability.

Another possible explanation is that the smaller cages of MOF-505 are inaccessible to the larger adsorbates, ethane and ethylene. This could explain why adsorption of those two gases is overestimated, while that of methane is not. However, this is unlikely because due to the channel-like nature of the pore topology, blocking the small cages would prevent access of the adsorbate molecules to the remainder of the pore space. Nevertheless, this hypothesis was tested by conducting a GCMC simulation of ethane for MOF-505 in which the small cages were blocked (further information on the blocking technique can be found in Figure S6). As can be seen in Figure 10 , this led to poor agreement between simulation and experiment. The removal of the small cages leads to a much lower initial uptake of ethane, as the strong van der Waals sites in the small cage are no longer available, and to a large underestimation of the adsorbent capacity.

The final possible hypothesis that was considered was incomplete equilibration of the experimental isotherms for both ethane and ethylene. This consideration was prompted by the rather small estimated pore limiting diameter of $4.53 \AA$ in MOF-505. As can be seen in Table 4, methane will likely be unaffected by the smaller window size, as all dimensions of the adsorbate are smaller than the pore limiting diameter. However, in the case of ethane, and therefore also ethylene, the adsorbate has one dimension that is longer than the pore limiting diameter. This means that those molecules need to find the correct orientation to diffuse through this constriction. As such, although both of those gases will still have full access to the entire pore volume of the MOF, it is likely that diffusion of ethane and ethylene will be much slower than that of methane, which raises questions as to whether the experimental measurements have truly reached equilibrium.

This hypothesis was again tested by partially blocking the pore space of the MOF in GCMC simulations, but in this case one small and one large cage of the unit cell were blocked to represent incomplete diffusion through the MOF in a simplified way. As illustrated in Figure 10, this led to very good agreement in ethane isotherms. Interestingly, the simulated isotherms with blocked pores could be replicated by simply scaling the original (unblocked) isotherms by a constant factor. This reflects the fact that we are simply restricting access to a portion of the pore volume, without specifically blocking one type of cage. Optimal agreement with experiments for ethane at both temperatures is obtained by scaling the original simulated isotherms by 0.8 , as shown in Figure 11a. The same scaling factor was then applied to ethylene, with results shown in Figure 11b. Although a systematic overestimation is still observed, this is now of the 


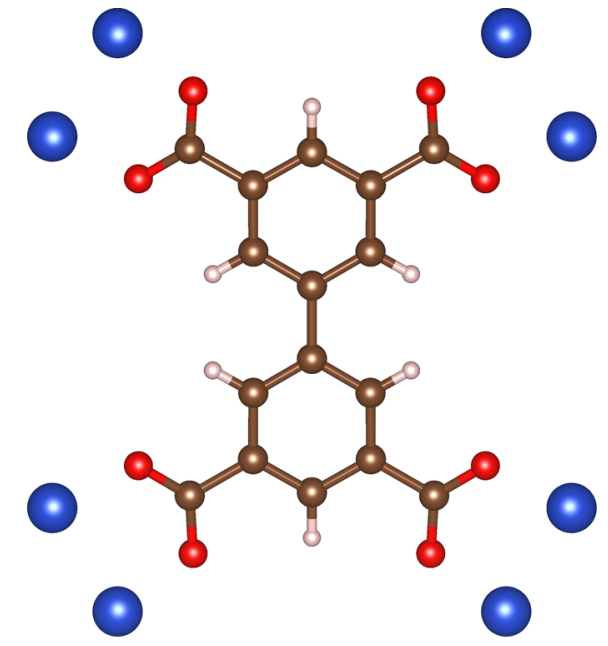

(a) Linker

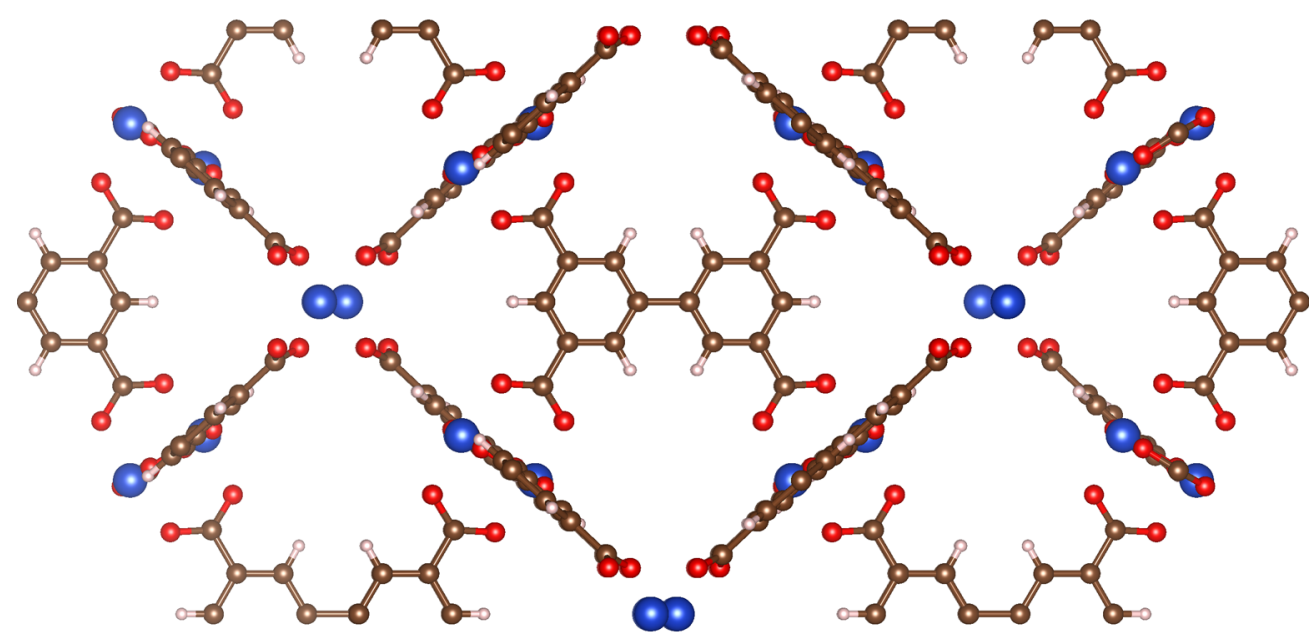

(b) Topology

Figure 7. Diagram showing the linker (a) and the unit cell topology (b) of MOF-505. Color code is the same as in Figure 1.

same magnitude as observed in the other MOFs of this family (compare the RMSDs in Table 5), reinforcing our argument that the discrepancies originally observed for ethane and ethylene have the same origin.

Although we believe our analysis presents a compelling case for incomplete experimental equilibration as the cause of the observed discrepancies for MOF-505, we cannot be certain of this without performing additional experiments. Nevertheless, further support for our hypothesis comes from recent adsorption experiments by Jiao et al. ${ }^{71}$ on NOTT-101 and a related material, ZJNU-80. Those authors have observed experimental isotherms with unusual curvature and strong hysteresis for several butene isomers, whereas such effects were absent in isotherms of small gases like $\mathrm{CO}_{2}, \mathrm{~N}_{2}$, and $\mathrm{CH}_{4}$, and were practically negligible for other $\mathrm{C} 4$ hydrocarbon isomers. Such a marked difference between chemically similar isomers is unlikely to arise from different adsorption energies, and is much more likely to be due to diffusional limitations experienced by some of the butene isomers. This is consistent with our interpretation of the MOF-505 results for ethane and ethylene, as the length of the butene isomer molecules is larger than the window size in NOTT-101, potentially leading to such diffusional limitations. Clearly, further work, both experimental and theoretical, is required to unequivocally clarify this issue.

3.5. UMCM-150. UMCM- $150^{72}$ is a much more complex MOF than those previously studied in this work and has the composition $\mathrm{Cu}_{3}(\text { bhtc) })_{2}$, where (bhtc) is biphenyl-3,4',5tricarboxylate. $^{62}$ This MOF is made up of two different types of inorganic building units. The first is the standard copper dimer paddlewheel MOF; however, there is also a copper trimer unit, $^{62,72}$ as shown in Figure $12 \mathrm{~b}$, which also exhibits CUS. The two types of metal building units are joined using an asymmetrically substituted linker molecule, as shown in Figure $12 \mathrm{a}, \mathrm{c}$, leading to three cage types: ${ }^{72}$ one large trigonal bipyramidal cage with dimensions $10.2 \times 13.7 \AA$, one smaller hexagonal bipyramidal cage with dimensions $5.2 \times 5.8 \AA$, and finally a small cylindrical cage with a diameter of $4.2 \AA^{62}$ The CUS interaction sites are facing into the two larger cages but not accessible from the smallest cage. Poreblazer identified the pore limiting diameter as $8.8 \AA$ but only found one dimension for percolation in what should be a $3 \mathrm{D}$ pore system (see Table S1). This indicates that the percolation path does not fully explore all of the cages but flows through the largest cages. Therefore, this large pore limiting diameter does not guarantee 

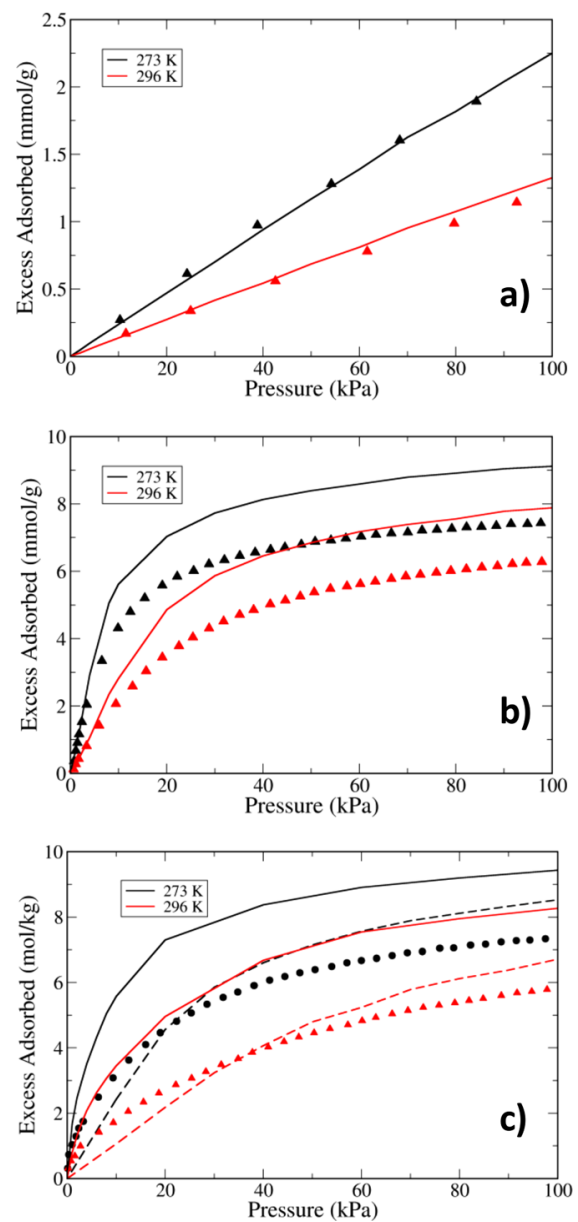

Figure 8. Simulated (lines) and experimental ${ }^{31}$ (points) adsorption isotherms in MOF-505 for (a) methane, (b) ethane, and (c) ethylene. For ethylene, the solid line shows results of the periodic DFT model, and the dashed line is for the standard LJ model (without CUS interaction).

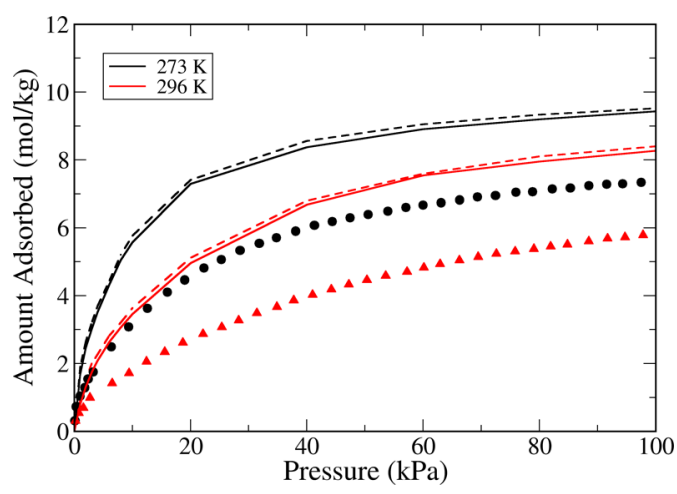

Figure 9. Comparison between simulated (lines) and experimental ${ }^{31}$ (points) ethylene adsorption isotherms in MOF-505. The solid line shows results from the original HKUST-1 parameters, while the dashed line shows results using new parameters obtained from DFT calculations on MOF-505.

that the adsorbates have full access to the smaller cages, as unlike in the previously investigated MOFs, diffusion through the large pores does not require access to the smaller cages.

The isotherms in Figure 13a,b show that simulations accurately capture both methane and ethane adsorption, suggesting that the entire pore volume of UMCM-150 is

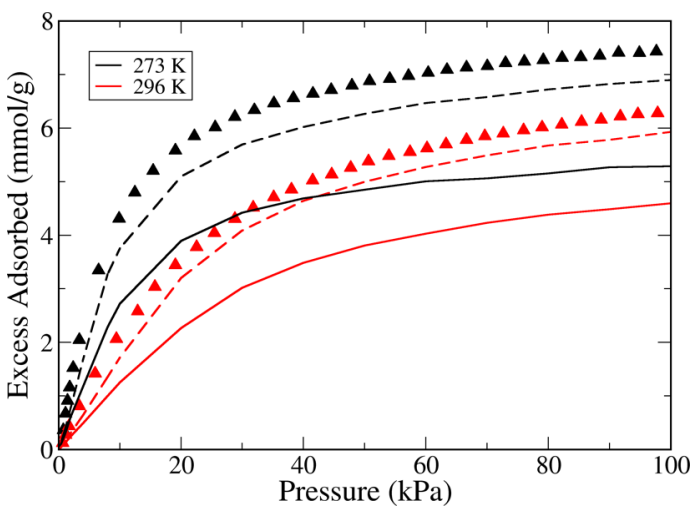

Figure 10. Comparison between simulated (lines) and experimental ${ }^{31}$ (points) ethane adsorption isotherms in MOF-505. The solid line shows results from simulations with all small cages blocked, while the dashed line shows results from simulations with one large and one small cage blocked.
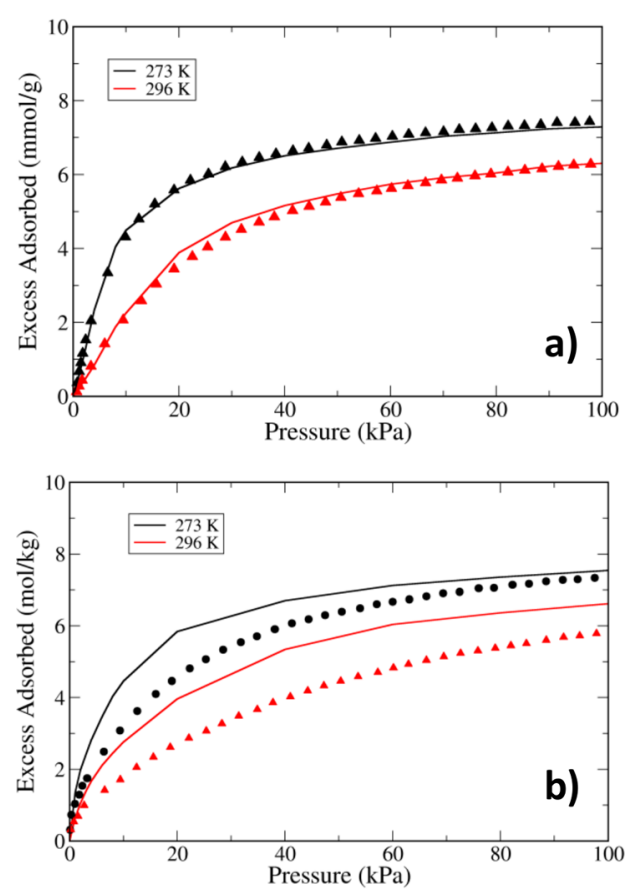

Figure 11. Simulated (lines) and experimental ${ }^{31}$ (points) adsorption isotherms in MOF-505 for (a) ethane and (b) ethylene. Simulations are scaled by a factor of 0.8 to represent incomplete equilibration of the experiments.

indeed accessible to both adsorbents (and hence also to ethylene). In our initial attempt to describe adsorption of ethylene on the CUS of this material, we have assumed that all metal sites (both in the dimer paddlewheel and in the trimer SBU) behaved equivalently. This assumption amounts to using the parameters obtained in the HKUST-1 paddlewheel for both types of CUS. In Figure 13c we can see that the new model simulation overestimates adsorption by a greater degree than observed in NOTT-101 and PCN-16 (the RMSD for ethylene on this MOF, shown in Table 5, is significantly higher than for the other MOFs), although it is worth noting that it still greatly improves agreement in comparison to the standard LJ model. We have conjectured that the reason for the additional discrepancies lies in the identical treatment of both copper building units using parameters fitted for only copper dimers. 


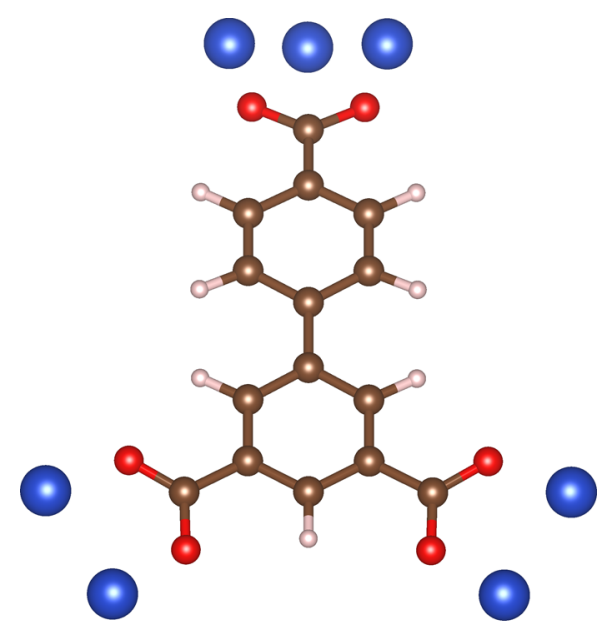

(a) Linker

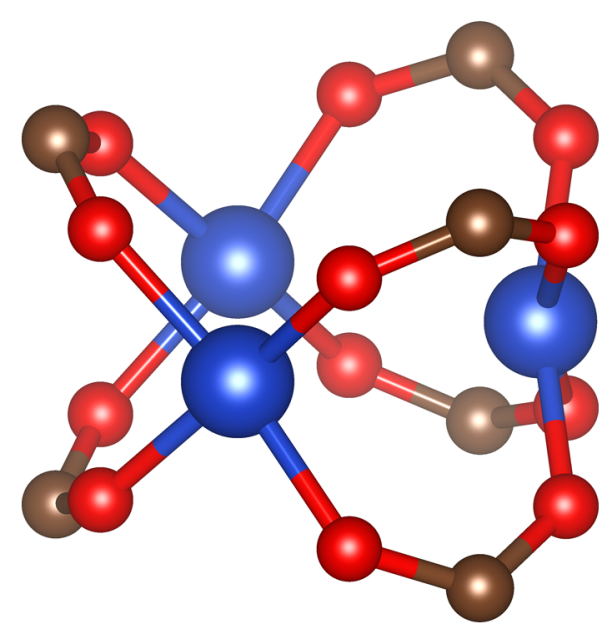

(b) Copper Trimer Building Unit

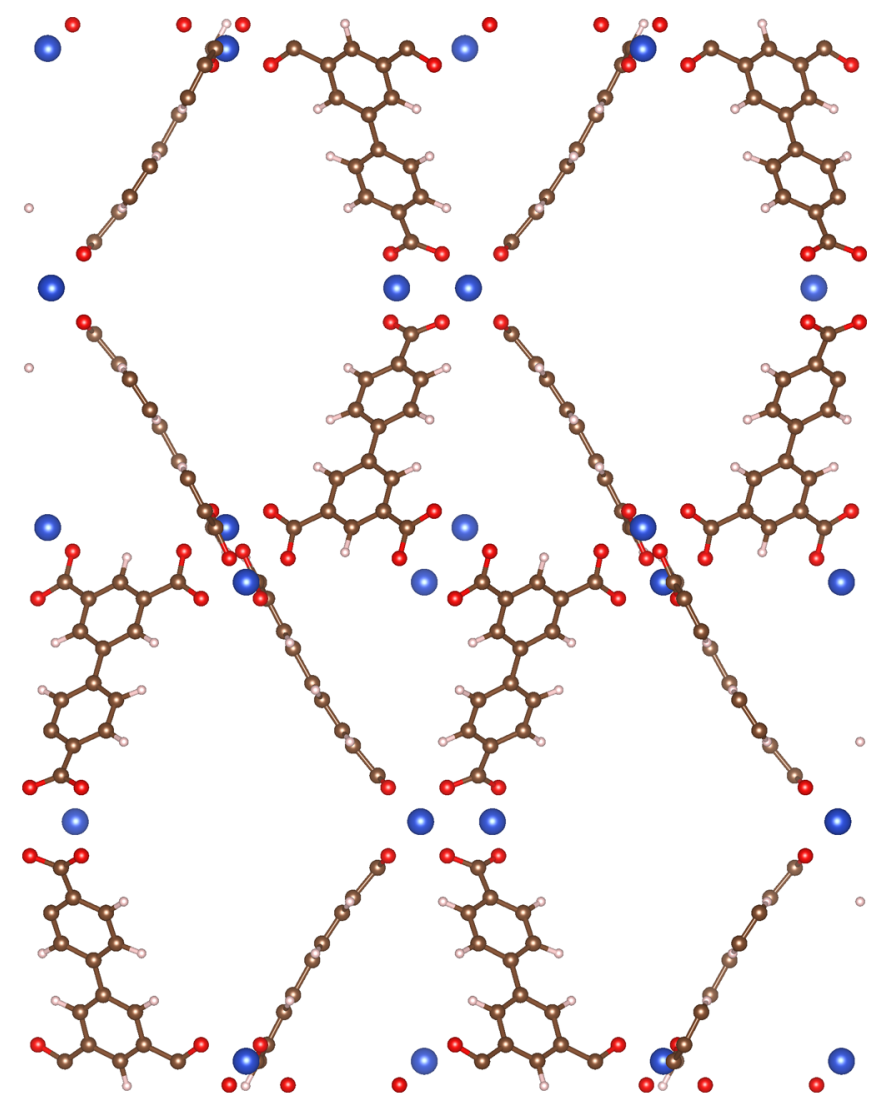

(c) Topology

Figure 12. Diagram showing the linker (a) and the unit cell topology (c) of UMCM-150, as well as a detailed view of the Cu-trimer metal center (b). Color code is the same as in Figure 1.

Initial DFT calculations indeed indicated that this is likely the cause, as the optimized DFT interaction energy between ethylene and an unsaturated $\mathrm{Cu}$ atom in the trimer was found to be $\sim-11 \mathrm{~kJ} / \mathrm{mol}$ at a binding distance of $3.1 \AA$. This is much less attractive than previously recorded for the paddlewheel dimer in both HKUST-1 and MOF-505, for which the DFT interaction energy is $\sim-23 \mathrm{~kJ} / \mathrm{mol}$ and the binding distance is 2.6 A. As such, a full DFT profile and new CUS parameters were obtained for the copper trimer of UMCM-150 using the procedure outlined in the Methodology. As can be seen in Figure 14, treating the metal building units independently leads to better agreement between simulation and experiment. Although a systematic overestimation is still observed, this is now of the same order as in all of the previously studied MOFs (see Table 5). Additionally, we have found that turning off the CUS interaction completely for the copper trimer leads to very similar simulation results as obtained by treating the trimer independently (see Figure S8). This therefore indicates that the 

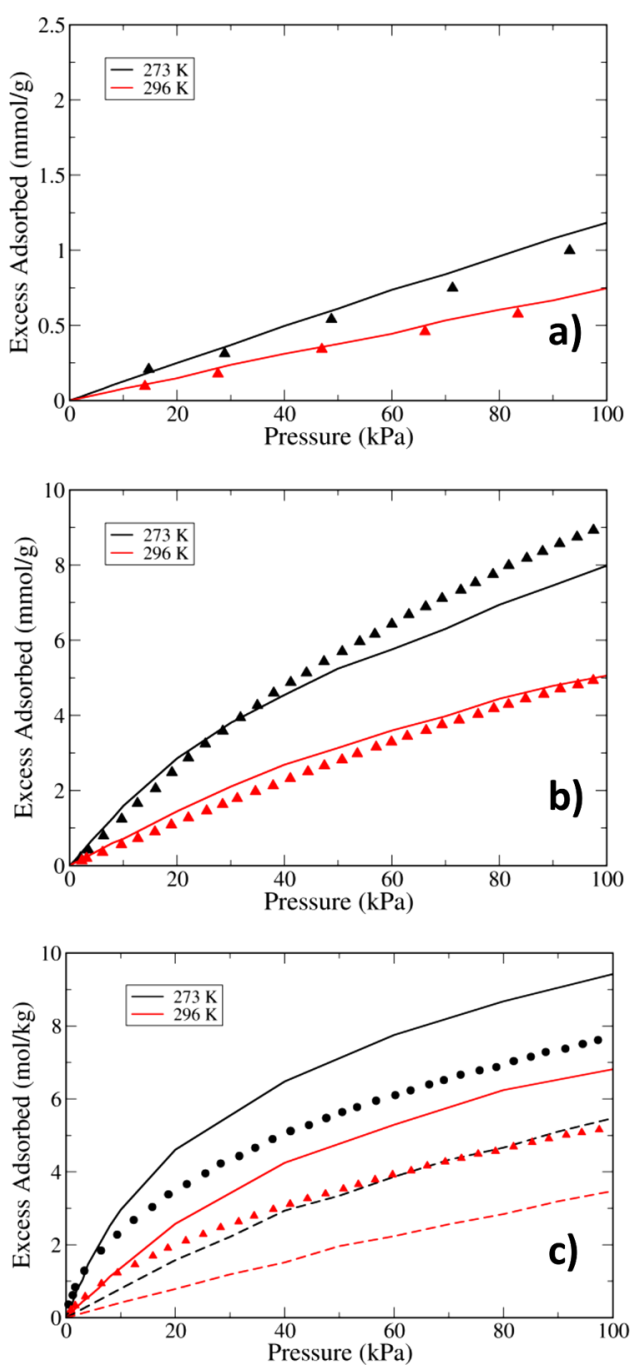

Figure 13. Simulated (lines) and experimental ${ }^{31}$ (points) adsorption isotherms in UMCM-150 for (a) methane, (b) ethane, and (c) ethylene. For ethylene, the solid line shows results of the periodic DFT model, and the dashed line is for the standard LJ model (without CUS interaction).

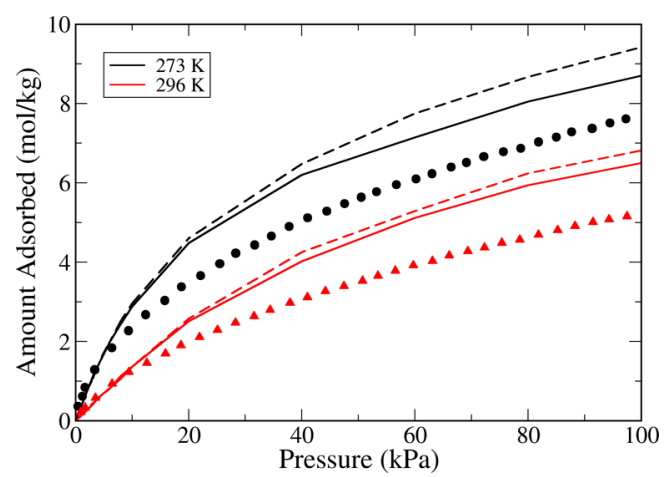

Figure 14. Comparison between simulated (lines) and experimental ${ }^{31}$ (points) ethylene adsorption isotherms in UMCM-150. The solid line shows results using separate CUS parameters for $\mathrm{Cu}$ dimers and trimers, while the dashed line shows results obtained considering all $\mathrm{Cu}$ atoms to behave equivalently.

role of the copper trimer CUS interaction in ethylene adsorption is relatively small at the considered temperatures, and therefore can potentially be ignored. However, ignoring this interaction on the basis of a comparison to experimental isotherms may lead to an incorrect distribution of adsorbed molecules, which may have a measurable impact on purecomponent or mixture adsorption at different thermodynamic conditions.

3.6. UTSA-20. UTSA $-20^{73}$ has the composition $\mathrm{Cu}_{3}(\mathrm{BHB})$ and an overall topology of type $z y g .^{73}$ The MOF is made up through self-assembly of a very large organic hexacarboxylate linker $\mathrm{BHB}\left(\mathrm{BHB}=3,3^{\prime}, 3^{\prime \prime}, 5,5^{\prime}, 5^{\prime \prime}\right.$-benzene-1,3,5-triylhexabenzoate), shown in Figure 15a, and copper paddlewheel as the inorganic building block. ${ }^{73}$ The complex linker group leads to two pore types, a rectangular pore with dimensions $3.4 \times 4.8 \AA$ and a cylindrical pore with a diameter of $8.5 \AA^{73}$ As can be seen in Figure 15b, this MOF has a very high density of CUS, which makes it an interesting candidate to further test the new CUS model. The CUS sites point toward, and therefore will be accessible from, both cage types. The presence of a smaller pore again raises questions about adsorbate accessibility to the full MOF structure. Similarly to UMCM-150, this complex structure also does not require diffusion through the small cages to access the larger cages; therefore, the pore limiting diameter found by Poreblazer was $8.8 \AA$ and percolation was in one dimension.

In Figure 16, it can be seen that simulations fail to describe experimental adsorption for all three adsorbates. In fact, this is the only MOF we studied where methane simulations are not correctly predicted. The fact that adsorption of all hydrocarbons, both saturated and unsaturated, is overestimated suggests that this is not related to the CUS model. Also, as methane is roughly spherical, it would be expected to diffuse relatively quickly through the framework; therefore, insufficient experimental equilibration is not a likely explanation for the observed discrepancies. Instead, the consistent overestimation may indicate that parts of the MOF (most likely the small rectangular cages) are not fully accessible to the three adsorbates. Furthermore, as Poreblazer identified percolation in only one dimension, with a large pore limiting diameter, this suggests molecules are able to diffuse through the MOF system using only the large pores, and therefore inaccessible small pores would not prevent or hinder diffusion in experiments.

Simulations with inaccessible cages were again conducted using the pore blocking technique outlined in Figure S7. The small cages and windows to these cages were blocked, and GCMC simulations were performed on the new structure. In Figure 17, it can be seen that making the small cages inaccessible in the GCMC simulations greatly improves agreement with experiment for all adsorbates. Nevertheless, agreement is not perfect, especially for ethylene for which there is a slight underestimation in the low pressure region. Our blocking approach is simply based on excluded volume, whereas in reality the cages will most likely be blocked by occluded solvent or reactant molecules. It has been shown that these occluded molecules can actually act as extra adsorption sites, and thus slightly enhance adsorption at low pressures, ${ }^{74,75}$ which could potentially explain the differences observed in Figure $17 \mathrm{c}$. More detailed studies are needed to fully clarify this issue, but this is outside the scope of this work. The main point we wish to make is that, whatever the root cause of the differences between simulation and experiment for UTSA-20, they are not due to the assumption of transferable parameters from the HKUST-1 paddlewheel. 


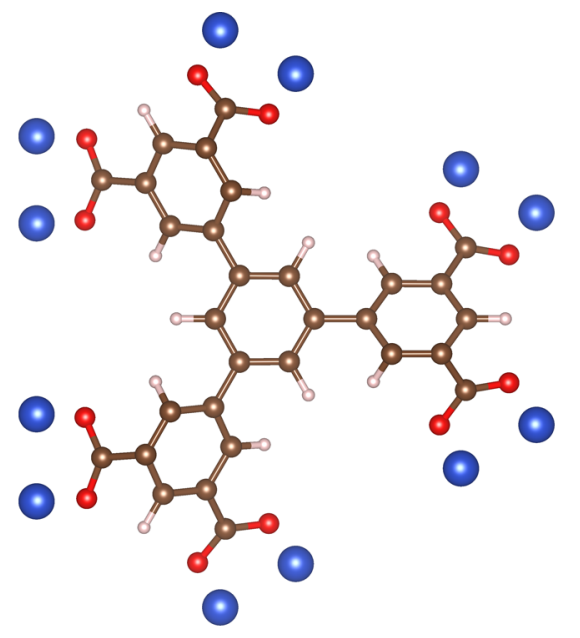

(a) Linker

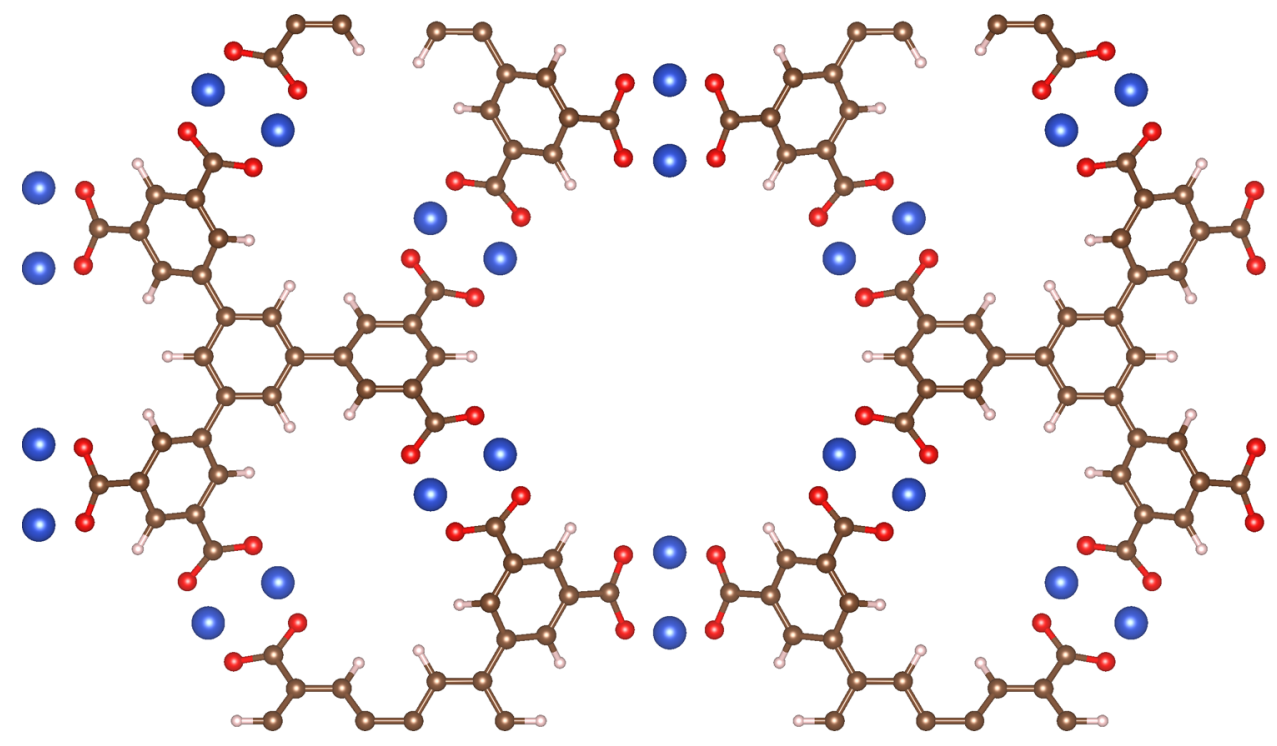

(b) Topology

Figure 15. Diagram showing the linker (a) and the unit cell topology (b) of UTSA-20. Color code is the same as in Figure 1.

\section{CONCLUSION}

In this Article, we build upon our computational approach for describing the complex orbital interactions present for some adsorbates in MOFs containing unsaturated metal sites. Using DFT-derived parameters for ethylene adsorption on a single MOF material (HKUST-1), we were able to obtain very good agreement with experimental isotherms on $\mathrm{PCN}-16$ and NOTT-101. Adsorption of both ethylene and ethane on MOF-505 was overestimated, but a possible explanation for this is insufficient equilibration of the experimental measurements. When this was accounted for in an approximate way, the same level of agreement as in the other MOFs was retrieved. In the case of UMCM-150, two different types of CUS sites were identified. We have shown that the CUS parameters were not transferable from a $\mathrm{Cu}$ paddlewheel unit to a $\mathrm{Cu}$ trimer unit, and that the latter required potential parameters to be separately determined. Using a correct description of the adsorption energy at this new site, good agreement with experiment was once again observed. Finally, adsorption of methane, ethane, and ethylene on UTSA-20 was consistently overestimated by roughly the same amount. We have proposed a reasonable explanation for this observation, based on inaccessible regions of the pore network, but additional work is needed to fully clarify this issue.

Our model was already shown to be transferable across different hydrocarbon adsorbates (e.g., ethylene to propylene), ${ }^{24}$ but in this work we have clearly demonstrated that it is in fact also transferable between different adsorbents of the same class. This is a key step in the development of a model capable of accurately predicting adsorption in CUS-containing MOFs for practical applications, including large-scale computational screening. We believe that the key for the success of our model lies in the decomposition of the DFT energy profiles to isolate the contribution of the $\mathrm{Cu}-\pi$ interaction and in a careful consideration of the local nature of this interaction. This was achieved through the addition of a new interaction site at the center of the olefin double bond, together with a cutoff scheme that prevents the metal site from interacting with more than one adsorbate molecule at the same time. In a recent paper, Kulkarni and Sholl $^{29}$ presented an alternative approach that 

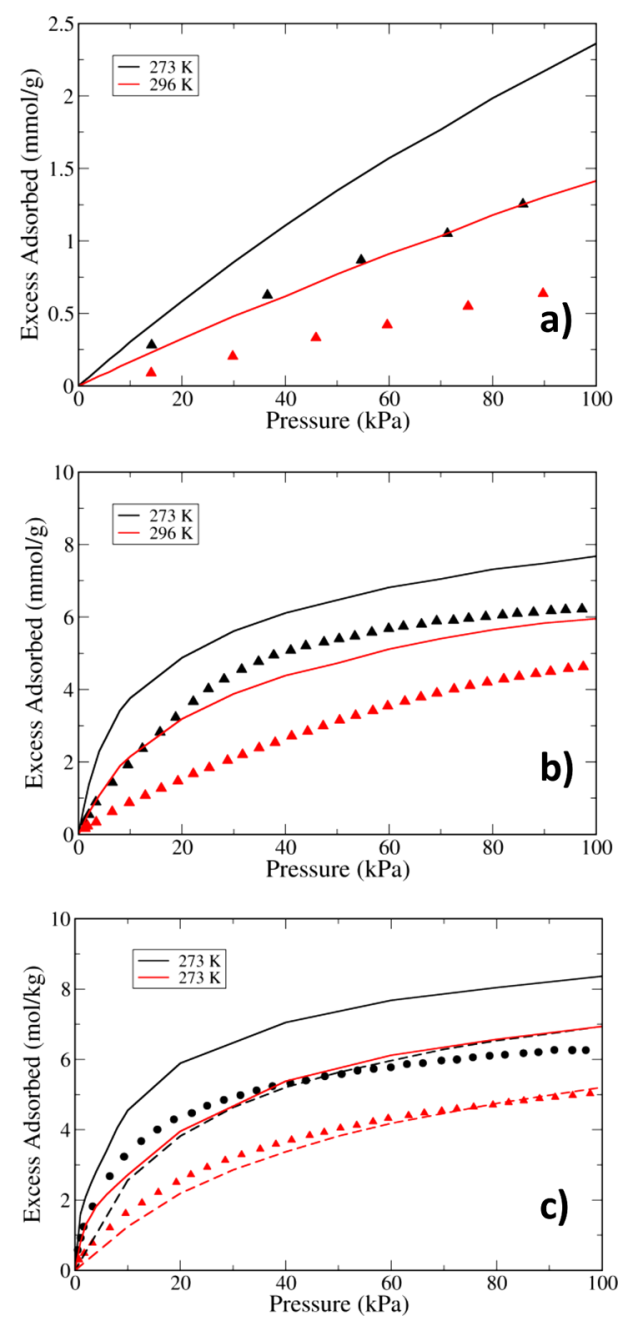

Figure 16. Simulated (lines) and experimental ${ }^{31}$ (points) adsorption isotherms in UTSA-20 for (a) methane, (b) ethane, and (c) ethylene. For ethylene, the solid line shows results of the periodic DFT model, and the dashed line is for the standard LJ model (without CUS interaction).

required the addition of a three-body term to avoid unreasonable adsorbate configurations. Their predictions of pure-component adsorption isotherms in the same series of MOFs studied here (see Figure S15 of their paper) were not as accurate as ours. Although more detailed analysis of their simulations is required, we speculate that the reason for the observed significant overestimation of ethylene adsorption in their paper may be due to the longer range of their CUS potential function, which may be allowing for the metal site to interact with more than one adsorbate molecule.

The success of our modeling approach opens the opportunity to develop new CUS parameters for different adsorbates and for accurately simulating competitive binary adsorption across a variety of systems, which is difficult to measure experimentally. In the future, we intend to expand our model to other gases such as $\mathrm{CO}_{2},{ }^{30}$ introducing the additional complexity of a significant electrostatic contribution to the adsorption energy. We believe that by treating the CUS interaction independently from all other intermolecular forces, it places our model in a prime position to respond quickly to any improvements in the description of these intermolecular forces.
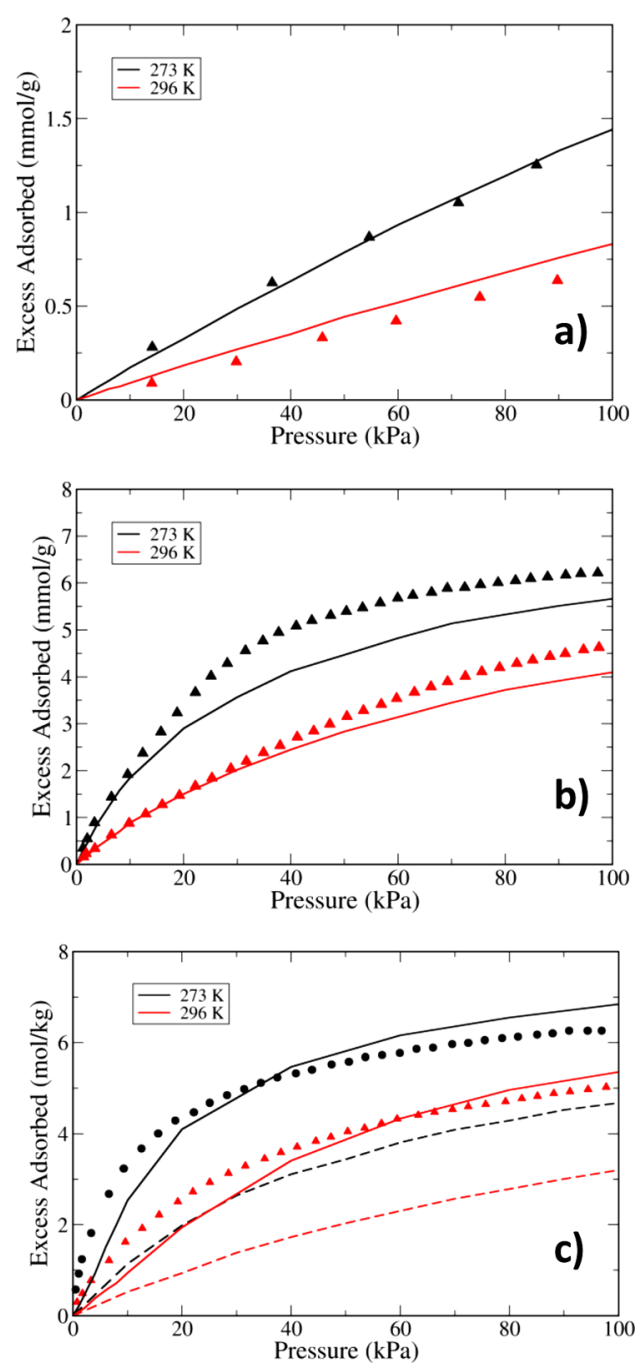

Figure 17. Simulated (lines) and experimental ${ }^{31}$ (points) adsorption isotherms in UTSA-20 with all small cages blocked for (a) methane, (b) ethane, and (c) ethylene. For ethylene, the solid line shows results of the periodic DFT model, and the dashed line is for the standard LJ model (without CUS interaction).

\section{ASSOCIATED CONTENT}

\section{Supporting Information}

The Supporting Information is available free of charge on the ACS Publications website at DOI: 10.1021/acs.jpcc.6b10751.

CUS interaction cutoff scheme, isolation of CUS interaction, profiles for ethylene interaction with HKUST-1 CUS, Poreblazer analysis, description of GCMC blocking procedure, CUS interaction for UMCM-150 trimer, description of root-mean-square deviation calculations, and seven figures and two tables (PDF)

\section{AUTHOR INFORMATION}

\section{Corresponding Authors}

*E-mail: c.campbell@strath.ac.uk.

*E-mail: miguel.jorge@strath.ac.uk.

ORCID

Miguel Jorge: 0000-0003-3009-4725

Notes

The authors declare no competing financial interest. 


\section{ACKNOWLEDGMENTS}

We thank A. Fletcher and L. Sarkisov for their insightful discussions. C.C. thanks EPSRC for a doctoral training grant (EP/M506643/1). A visit of C.C. to Bremen was funded by the MAPEX Center for Materials and Processes, University of Bremen, through a Ph.D. Research Grant. M.F. is funded by the Central Research Development Fund (CRDF) of the University of Bremen (Funding Line 04- Independent Projects for Post-Docs). M.J. and C.A.F.-R. are thankful for funding from EPSRC UK Project Grant EP/L014297/1. J.R.B.G. is thankful to FCT/MEC and FEDER for projects CICECOAveiro Institute of Materials, POCI-01-0145-FEDER-007679 (FCT ref. UID/CTM/50011/2013), and Investigador FCT.

\section{REFERENCES}

(1) Zhou, H.-C.; Long, J. R.; Yaghi, O. M. Introduction to MetalOrganic Frameworks. Chem. Rev. 2012, 112, 673-674.

(2) CCDC, How Many MOFs Are There In The CSD. 2014; https://www.ccdc.cam.ac.uk/support-and-resources/support/case/ ?caseid/9833bd2c-27f9-4ff7-8186-71a9b415f012, last accessed 16 August 2016.

(3) O’Keeffe, M.; Ockwig, N. W.; Chae, H. K.; Eddaoudi, M.; Kim, J.; Yaghi, O. M. Reticular Synthesis and the Design of New Materials. Nature 2003, 423, 705-714.

(4) Wilmer, C. E.; Leaf, M.; Lee, C. Y.; Farha, O. K.; Hauser, B. G.; Hupp, J. T.; Snurr, R. Q. Large-scale Screening of Hypothetical MetalOrganic Frameworks. Nat. Chem. 2011, 4, 83-89.

(5) Arstad, B.; Fjellvåg, H.; Kongshaug, K. O.; Swang, O.; Blom, R. Amine Functionalised Metal Organic Frameworks (MOFs) as Adsorbents for Carbon Dioxide. Adsorption 2008, 14, 755-762.

(6) Eddaoudi, M.; Kim, J.; Rosi, N.; Vodak, D.; Wachter, J.; O'Keeffe, M.; Yaghi, O. M. Systematic Design of Pore Size and Functionality in Isoreticular MOFs and Their Application in Methane Storage. Science 2002, 295, 469-472.

(7) Nugent, P.; Belmabkhout, Y.; Burd, S. D.; Cairns, A. J.; Luebke, R.; Forrest, K.; Pham, T.; Ma, S.; Space, B.; Wojtas, L.; Eddaoudi, M.; Zaworotko, M. J. Porous Materials with Optimal Adsorption Thermodynamics and Kinetics for $\mathrm{CO}_{2}$ Separation. Nature 2013, $495,80-84$.

(8) Watanabe, T.; Sholl, D. S. Accelerating Applications of MetalOrganic Frameworks for Gas Adsorption and Separation by Computational Screening of Materials. Langmuir 2012, 28, 1411414128.

(9) Fischer, M.; Gomes, J. R.; Jorge, M. Computational Approaches to Study Adsorption in MOFs with Unsaturated Metal Sites. Mol. Simul. 2014, 40, 537-556.

(10) Keskin, S.; Liu, J.; Rankin, R. B.; Johnson, J. K.; Sholl, D. S. Progress, Opportunities, and Challenges for Applying Atomically Detailed Modeling to Molecular Adsorption and Transport in MetalOrganic Framework Materials. Ind. Eng. Chem. Res. 2009, 48, 23552371.

(11) Yang, Q.; Zhong, C. Molecular Simulation of Adsorption and Diffusion of Hydrogen in Metal-Organic Frameworks. J. Phys. Chem. B 2005, 109, 11862-11864.

(12) Uzun, A.; Keskin, S. Site Characteristics in Metal Organic Frameworks for Gas Adsorption. Prog. Surf. Sci. 2014, 89, 56-79.

(13) Liu, Y.; Liu, J.; Lin, Y. Strong Binding Site Molarity of MOFs and its Effect on $\mathrm{CO}_{2}$ Adsorption. Microporous Mesoporous Mater. 2015, 214, 242-245.

(14) Zhang, Y.; Li, B.; Krishna, R.; Wu, Z.; Ma, D.; Shi, Z.; Pham, T.; Forrest, K.; Space, B.; Ma, S. Highly Selective Adsorption of Ethylene Over Ethane in a MOF Featuring the Combination of Open Metal Site and $\pi$-Complexation. Chem. Commun. 2015, 51, 2714-2717.

(15) Dincă, M.; Dailly, A.; Liu, Y.; Brown, C. M.; Neumann, D. A.; Long, J. R. Hydrogen Storage in a Microporous Metal-Organic Framework with Exposed Mn2+ Coordination Sites. J. Am. Chem. Soc. 2006, 128, 16876-16883.
(16) Grande, C. A.; Rodrigues, A. E. Propane/Propylene Separation by Pressure Swing Adsorption Using Zeolite 4A. Ind. Eng. Chem. Res. 2005, 44, 8815-8829.

(17) Dewar, J. S. A review of the pi-Complex Theory. Bull. Soc. Chim. Fr. 1951, 18, C71-C19.

(18) Chatt, J.; Duncanson, L. A. Olefin Co-ordination Compounds. Part III. Infra-red Spectra and Structure: Attempted Preparation of Acetylene Complexes. J. Chem. Soc. 1953, 2939-2947.

(19) Nechaev, M. S.; Rayón, V. M.; Frenking, G. Energy Partitioning Analysis of the Bonding in Ethylene and Acetylene Complexes of Group 6, 8, and 11 Metals: $(\mathrm{CO})_{5} \mathrm{TM}-\mathrm{C}_{2} \mathrm{H}_{x}$ and $\mathrm{Cl}_{4} \mathrm{TM}-\mathrm{C}_{2} \mathrm{H}_{x}(\mathrm{TM}=$ $\mathrm{Cr}, \mathrm{Mo}, \mathrm{W}),(\mathrm{CO})_{4} \mathrm{TM}-\mathrm{C}_{2} \mathrm{H}_{x}(\mathrm{TM}=\mathrm{Fe}, \mathrm{Ru}, \mathrm{Os})$, and $\mathrm{TM}+-\mathrm{C}_{2} \mathrm{H}_{x}$ $(\mathrm{TM}=\mathrm{Cu}, \mathrm{Ag}, \mathrm{Au}) . J$. Phys. Chem. A 2004, 108, 3134-3142.

(20) Lamia, N.; Jorge, M.; Granato, M. A.; Paz, F. A. A.; Chevreau, H.; Rodrigues, A. E. Adsorption of Propane, Propylene and Isobutane on a Metal-Organic Framework: Molecular Simulation and Experiment. Chem. Eng. Sci. 2009, 64, 3246-3259.

(21) Jorge, M.; Lamia, N.; Rodrigues, A. E. Molecular Simulation of Propane/Propylene Separation on the Metal-Organic Framework CuBTC. Colloids Surf., A 2010, 357, 27-34.

(22) Verma, P.; Xu, X.; Truhlar, D. G. Adsorption on Fe-MOF-74 for C1-C3 Hydrocarbon Separation. J. Phys. Chem. C 2013, 117, 1264812660 .

(23) Castillo, J. M.; Vlugt, T. J. H.; Calero, S. Understanding Water Adsorption in Cu-BTC Metal-Organic Frameworks. J. Phys. Chem. C 2008, 112, 15934-15939.

(24) Jorge, M.; Fischer, M.; Gomes, J. R. B.; Siquet, C.; Santos, J. C.; Rodrigues, A. E. Accurate Model for Predicting Adsorption of Olefins and Paraffins on MOFs with Open Metal Sites. Ind. Eng. Chem. Res. 2014, 53, 15475-15487.

(25) Fischer, M.; Hoffmann, F.; Fröba, M. Metal-Organic Frameworks and Related Materials for Hydrogen Purification: Interplay of Pore Size and Pore Wall Polarity. RSC Adv. 2012, 2, 4382-4396.

(26) Chen, L.; Grajciar, L.; Nachtigall, P.; Düren, T. Accurate Prediction of Methane Adsorption in a Metal-Organic Framework with Unsaturated Metal Sites by Direct Implementation of an ab Initio Derived Potential Energy Surface in GCMC Simulation. J. Phys. Chem. C 2011, 115, 23074-23080.

(27) Dzubak, A. L.; Lin, L.-C.; Kim, J.; Swisher, J. A.; Poloni, R.; Maximoff, S. N.; Smit, B.; Gagliardi, L. Ab Initio Carbon Capture in Open-Site Metal-Organic Frameworks. Nat. Chem. 2012, 4, 810-816.

(28) Fischer, M.; Gomes, J. R. B.; Fröba, M.; Jorge, M. Modeling Adsorption in Metal-Organic Frameworks with Open Metal Sites: Propane/Propylene Separations. Langmuir 2012, 28, 8537-8549.

(29) Kulkarni, A. R.; Sholl, D. S. Screening of Copper Open Metal Site MOFs for Olefin/Paraffin Separations Using DFT-Derived Force Fields. J. Phys. Chem. C 2016, 120, 23044-23054.

(30) Fischer, M.; Hoffmann, F.; Fröba, M. New Microporous Materials for Acetylene Storage and $\mathrm{C}_{2} \mathrm{H}_{2} / \mathrm{CO}_{2}$ Separation: Insights from Molecular Simulations. ChemPhysChem 2010, 11, 2220-2229.

(31) He, Y.; Krishna, R.; Chen, B. Metal-organic Frameworks with Potential for Energy-efficient Adsorptive Separation of Light Hydrocarbons. Energy Environ. Sci. 2012, 5, 9107-9120.

(32) Bloch, E. D.; Queen, W. L.; Krishna, R.; Zadrozny, J. M.; Brown, C. M.; Long, J. R. Hydrocarbon Separations in a Metal-Organic Framework with Open Iron(II) Coordination Sites. Science 2012, 335, $1606-1610$

(33) Hutter, J.; Iannuzzi, M.; Schiffmann, F.; VandeVondele, J. CP2K: atomistic simulations of condensed matter systems. Wiley Interdisciplinary Reviews: Computational Molecular Science 2014, 4, 1525.

(34) Hartwigsen, C.; Goedecker, S.; Hutter, J. Relativistic Separable Dual-space Gaussian Pseudopotentials from $\mathrm{H}$ to Rn. Phys. Rev. B 1998, 58, 3641-3662.

(35) Perdew, J. P.; Burke, K.; Ernzerhof, M. Generalized Gradient Approximation Made Simple. Phys. Rev. Lett. 1996, 77, 3865-3868.

(36) Fischer, M.; Kuchta, B.; Firlej, L.; Hoffmann, F.; Fröba, M. Accurate Prediction of Hydrogen Adsorption in Metal-Organic Frameworks with Unsaturated Metal Sites via a Combined Density- 
Functional Theory and Molecular Mechanics Approach. J. Phys. Chem. C 2010, 114, 19116-19126.

(37) Ramalho, J. P. P.; Gomes, J. R. B.; Illas, F. Accounting for van der Waals Interactions Between Adsorbates and Surfaces in Density Functional Theory Based Calculations: Selected Examples. RSC Adv. 2013, 3, 13085-13100.

(38) Tkatchenko, A.; von Lilienfeld, O. A. Adsorption of Ar on Graphite using London Dispersion Forces Corrected Kohn-Sham Density Functional Theory. Phys. Rev. B 2006, 73, 153406.

(39) Johnson, E. R.; Mackie, I. D.; DiLabio, G. A. Dispersion Interactions in Density-functional Theory. J. Phys. Org. Chem. 2009, 22, 1127-1135.

(40) Krack, M. Pseudopotentials for $\mathrm{H}$ to Kr Optimized for GradientCorrected Exchange-Correlation Functionals. Theor. Chem. Acc. 2005, 114, 145-152.

(41) Goedecker, S.; Teter, M.; Hutter, J. Separable Dual-space Gaussian Pseudopotentials. Phys. Rev. B: Condens. Matter Mater. Phys. 1996, 54, 1703-1710.

(42) Delley, B. An All-Electron Numerical Method for Solving the Local Density Functional for Polyatomic Molecules. J. Chem. Phys. 1990, 92, 508-517.

(43) Delley, B. From Molecules to Solids with the DMol3 Approach. J. Chem. Phys. 2000, 113, 7756-7764.

(44) Accelrys Software Inc., Material Studio Version 5, 2011; http:// accelrys.com/products/collaborative-science/biovia-materials-studio/.

(45) Weeks, J. D.; Chandler, D.; Andersen, H. C. Role of Repulsive Forces in Determining the Equilibrium Structure of Simple Liquids. J. Chem. Phys. 1971, 54, 5237-5247.

(46) Chandler, D.; Weeks, J. D.; Andersen, H. C. Van der Waals Picture of Liquids, Solids, and Phase Transformations. Science 1983, 220, 787-794.

(47) Gupta, A.; Chempath, S.; Sanborn, M. J.; Clark, L. A.; Snurr, R. Q. Object-oriented Programming Paradigms for Molecular Modeling. Mol. Simul. 2003, 29, 29-46.

(48) Snurr, R. Q.; Bell, A. T.; Theodorou, D. N. Prediction of Adsorption of Aromatic Hydrocarbons in Silicalite from Grand Canonical Monte Carlo Simulations with Biased Insertions. J. Phys. Chem. 1993, 97, 13742-13752.

(49) Peng, D. Y.; Robinson, D. B. A New Two-Constant Equation of State. Ind. Eng. Chem. Fundam. 1976, 15, 59-64.

(50) Myers, A. L.; Monson, P. A. Adsorption in Porous Materials at High Pressure: Theory and Experiment. Langmuir 2002, 18, 1026110273.

(51) Mayo, S. L.; Olafson, B. D.; Goddard, W. A. DREIDING: A Generic Force Field for Molecular Simulations. J. Phys. Chem. 1990, 94, 8897-8909.

(52) Düren, T.; Bae, Y.-S.; Snurr, R. Q. Using Molecular Simulation to Characterise Metal-Organic Frameworks for Adsorption Applications. Chem. Soc. Rev. 2009, 38, 1237-1247.

(53) Sarkisov, L.; Düren, T.; Snurr, R. Q. Molecular Modelling of Adsorption in Novel Nanoporous Metal-Organic Materials. Mol. Phys. 2004, 102, 211-221.

(54) Pillai, R. S.; Pinto, M. L.; Pires, J.; Jorge, M.; Gomes, J. R. B. Understanding Gas Adsorption Selectivity in IRMOF-8 Using Molecular Simulation. ACS Appl. Mater. Interfaces 2015, 7, 624-637.

(55) Rappe, A. K.; Casewit, C. J.; Colwell, K. S.; Goddard, W. A.; Skiff, W. M. UFF, A Full Periodic Table Force Field for Molecular Mechanics and Molecular Dynamics Simulations. J. Am. Chem. Soc. 1992, 114, 10024-10035.

(56) Martin, M. G.; Siepmann, J. I. Transferable Potentials for Phase Equilibria. 1. United- Atom Description of n-Alkanes. J. Phys. Chem. B 1998, 102, 2569-2577.

(57) Wick, C. D.; Martin, M. G.; Siepmann, J. I. Transferable Potentials for Phase Equilibria. 4. United-atom Description of Linear and Branched Alkenes and Alkylbenzenes. J. Phys. Chem. B 2000, 104, 8008-8016.

(58) Sarkisov, L.; Harrison, A. Computational Structure Characterisation Tools in Application to Ordered and Disordered Porous Materials. Mol. Simul. 2011, 37, 1248-1257.
(59) Chui, S. S. Y.; Lo, S. M.; Charmant, J. P. H.; Orpen, A. G.; Williams, I. D. A Chemically Functionalizable Nanoporous Material $\left[\mathrm{Cu}_{3}(\mathrm{TMA})_{2}\left(\mathrm{H}_{2} \mathrm{O}\right)_{3}\right]_{\mathrm{n}}$. Science 1999, 283, 1148-1150.

(60) DeCoste, J. B.; Peterson, G. W.; Schindler, B. J.; Killops, K. L.; Browe, M. A.; Mahle, J. J. The Effect of Water Adsorption on the Structure of the Carboxylate Containing Metal-Organic Frameworks Cu-BTC, Mg-MOF-74, and UiO-66. J. Mater. Chem. A 2013, 1, 11922-11932.

(61) Marx, S.; Kleist, W.; Baiker, A. Synthesis, Structural Properties, and Catalytic Behavior of Cu-BTC and Mixed-Linker Cu-BTC-PyDC in the Oxidation of Benzene Derivatives. J. Catal. 2011, 281, 76-87.

(62) Fischer, M. Molecular Simulations of Hydrogen Storage and Gas Separation in Metal-Organic Frameworks. Ph.D. Thesis, Universität Hamburg, 2011.

(63) Grajciar, L.; Wiersum, A. D.; Llewellyn, P. L.; Chang, J.-S.; Nachtigall, P. Understanding $\mathrm{CO}_{2}$ Adsorption in CuBTC MOF: Comparing Combined DFT-ab Initio Calculations with Microcalorimetry Experiments. J. Phys. Chem. C 2011, 115, 17925-17933.

(64) Webster, C. E.; Drago, R. S.; Zerner, M. C. Molecular Dimensions for Adsorptives. J. Am. Chem. Soc. 1998, 120, 5509-5516.

(65) Hu, Y.; Xiang, S.; Zhang, W.; Zhang, Z.; Wang, L.; Bai, J.; Chen, B. A new MOF-505 Analog Exhibiting High Acetylene Storage. Chem. Commun. 2009, 7551-7553.

(66) Sun, D.; Ma, S.; Simmons, J. M.; Li, J.-R.; Yuan, D.; Zhou, H.-C. An Unusual Case of Symmetry-Preserving Isomerism. Chem. Commun. 2010, 46, 1329-1331.

(67) Li, M.; Li, D.; O’Keeffe, M.; Yaghi, O. M. Topological Analysis of Metal-Organic Frameworks with Polytopic Linkers and/or Multiple Building Units and the Minimal Transitivity Principle. Chem. Rev. 2014, 114, 1343-1370.

(68) Simmons, J. M.; Wu, H.; Zhou, W.; Yildirim, T. Carbon Capture in Metal-Organic Frameworks-A Comparative Study. Energy Environ. Sci. 2011, 4, 2177-2185.

(69) Lin, X.; Jia, J.; Zhao, X.; Thomas, K. M.; Blake, A. J.; Walker, G. S.; Champness, N. R.; Hubberstey, P.; Schröder, M. High $\mathrm{H}_{2}$ Adsorption by Coordination-Framework Materials. Angew. Chem., Int. Ed. 2006, 45, 7358-7364.

(70) Chen, B.; Ockwig, N. W.; Millward, A. R.; Contreras, D. S.; Yaghi, O. M. High $\mathrm{H}_{2}$ Adsorption in a Microporous Metal-Organic Framework with Open Metal Sites. Angew. Chem. 2005, 117, 48234827.

(71) Jiao, J.; Liu, H.; Bai, D.; He, Y. A Chemically Cross-Linked NbO-Type Metal-Organic Framework: Cage or Window Partition? Inorg. Chem. 2016, 55, 3974-3979.

(72) Wong-Foy, A. G.; Lebel, O.; Matzger, A. J. Porous Crystal Derived from a Tricarboxylate Linker with Two Distinct Binding Motifs. J. Am. Chem. Soc. 2007, 129, 15740-15741.

(73) Guo, Z.; Wu, H.; Srinivas, G.; Zhou, Y.; Xiang, S.; Chen, Z.; Yang, Y.; Zhou, W.; O'Keeffe, M.; Chen, B. A Metal-Organic Framework with Optimized Open Metal Sites and Pore Spaces for High Methane Storage at Room Temperature. Angew. Chem., Int. Ed. 2011, 50, 3178-3181.

(74) Al-Janabi, N.; Fan, X.; Siperstein, F. R. Assessment of MOF's Quality: Quantifying Defect Content in Crystalline Porous Materials. J. Phys. Chem. Lett. 2016, 7, 1490-1494.

(75) Sarkisov, L. Molecular Simulation of Low Temperature Argon Adsorption in Several Models of IRMOF-1 with Defects and Structural Disorder. Dalton Trans. 2016, 45, 4203-4212.

\section{NOTE ADDED IN PROOF}

During the manuscript production stage, we became aware of a paper by Heinen et al. (DOI: 10.1002/chem.201603895) that proposed a model for ethylene adsorption on HKUST-1 based on an approach that shares some similarities with our own. However, the transferability of the model was not tested. 